(Physiologisches Laboratorium in Bonn.)

\title{
Ueber Oxydation im Warmblüter bei subnormalen Temperaturen.
}

\author{
Von
}

Dr. phil. Wilhelm Velten, pract. Arzt.

\author{
Hierzu Tafel VII.
}

Nachdem Herr Geh. Rath Professor Dr. Pflïger in der Abhandlung "Ueber Wärme und Oxydation der lebendigen Materie" (dieses Archiv, Bd. 18 p. 247) die zahlreichen Untersuchungen niedergelegt, die er behufs der Darlegung der Grundphaenomene der Oxydation in ihrer Abhängigkeit von der Temperatur vom Gesichtspunkte seiner Theorie anstellte, beauftragte derselbe mich, im Anschluss an die auf p. 303 und 304 jener Abhandlung aufgestellten Resultate, eine Reihe von Versuchen bei subnormalen Temperaturen an curarisirten Kaninchen vorzunehmen, um die Oxydationscurve bis weit unterhalb der Normaltemperatur festzustellen.

Zur Messung der Oxydation wurden die Thiere in den Respirationsapparat eingeschaltet, dessen sich Finkler und Oertmann bei ihren Untersuchungen bedienten, und den sie in ihrer Abhandlung „Ueber den Einfluss der Athemmechanik auf den Stoffwechsel" (dieses Archiv Bd. 14 p. 38) beschrieben haben. Dieser Apparat wurde in einfacher Form schon 1863 von Pflüger und Dohmen zur Unterhaltung der künstlichen Respiration bei Thieren verwandt, später von Röhrig-Zuntz und Zuntz für Sauerstoffathmung eingerichtet; die urspriinglichen Gummiventile wurden durch Muller'sche Kaliventile, das Wasserspirometer durch das Quecksilberspirometer ersetzt. Das Wesentliche des Apparats ist das Hunter'sche Doppelgebläse, mittelst dessen abwechselnd Sauerstoff in die Lungen getrieben und wieder ausgesaugt wird. Der Sauerstoffverbrauch wird an dem calibrirten Spirometer, aus welchem die Ballons des Hunter'schen Gebläses den Sauerstoff be- 
ziehen, direct abgelesen; die in der Kalilange der Müller'schen Ventile absorbirte Kohlensäure wird in dem Vacuum der Pflüger'schen Gaspumpe dureh Phosphorsäure ausgetrieben und mit Berücksichtigung der ursprünglich in der Kalilauge schon vor dem Versuche enthaltenen volumenometrisch bestimmt. Alle gefundenen Werthe sind immer auf $0^{\circ}$ und $0,76 \mathrm{~m}$ Quecksilber reducirt, und beziehen sich immer auf 1 Kilo Thiergewicht und 1 Stunde Zeit. Wegen der sehr zahlreichen Gummischläuche, die vor ihrer Anbringung immer unter Wasserdruck geprift wurden, wurde der Apparat häufig auf seinen hermetischen Verschluss untersucht und etwaige Lecke nach oft recht mühsamem Suchen entdeckt und entfernt.

Da es sich um Untersuchungen bei tieferen Temperaturen handelte, so wurden zur Vermeidung von Schwankungen in der umgebenden Luft sämmtliche Versuchsreihen bei Winterkälte in einem im Souterrain des physiologischen Instituts gelegenen ungeheizten Raume ausgeführt. Die Vorbereitungen waren dieselben bei allen Versuchsreihen; nachdem das Thier auf einem Kaninchenhalter befestigt war, wurde die Tracheotomie gemacht und die dreizackige Tracheacanïle des Respirationsapparats in die Trachea des Thieres mit doppelter Ligatur eingebunden. Das Thier athmete so lange durch einen Seitenschlauch aus der freien Luft, bis die eintretende Curarenarkose die künstliche Athmung, zuerst mittelst eines Blasebalges bis zum Beginn des Versuchs, dann mittelst des Apparates selbst nöthig machte. Das Thier wurde durchschnittlich 1/2 Stunde vor Beginn des ersten Versuches curarisirt, bald darauf in ein Bad mit bestimmter Temperatur versenkt, so dass sich nur noch Kopf und Brust des Kaninchens ausserhalb des Wassers befanden. Die Temperatur des Bades regulirte die des Thieres; letztere wurde an einem in Recto liegenden, ZehntelGrade zeigenden Thermometer fortwährend beobachtet und notirt.

Wie bei den von Pflüger angestellten Versuchen waren auch hier trotz der in der Curarenarkose gesunkenen Energie der Oxydation die Herzpulsationen kräftig, die Arterien strotzend mit hellrothem Blute erfiullt, auch das Venenblut erschien heller, so dass von einer Störung des Kreislaufes und behinderten Circulation, oder verminderter Zufuhr von Sauerstoff zu den Organen auch in diesen Versuchen die Verringerung der Oxydation nicht abgeleitet werden kann. 
Ueber Oxydation im Warmblüter bei subnormalen Temperaturen. 363

\section{Serie I (21. Nov.)}

Kaninchen von $1624 \mathrm{gr}$ curarisirt. Es wurden circa 68 künstliche Athemzüge mittelst der Gummiballons hervorgebracht.

\section{Versuch I.}

Anfang des Versuches 1 Uhr 28 Min. Dauer 20 Min.

$$
\begin{gathered}
\text { Temperatur: des Thieres } 38^{\circ}, 4 \mathrm{C} \text {. } \\
\text { des Bades } 37^{\circ}, 0 \mathrm{C} \text {. }
\end{gathered}
$$

a) Anfangsvolum auf $0^{\circ} \mathrm{C}$. und $760 \mathrm{~mm}$ reducirt . . . $=682,07 \mathrm{~cm}$

b) Endvolum auf $0^{\circ} \mathrm{C}$. und $760 \mathrm{~mm}$ reducirt . . . . = 348,23" Also Sauerstoffverbrauch in $20 \mathrm{Min}$. . . . . . . . = 333,84 "

Hieraus folgt für 1 Kilo and 1 Stunde ein Sanerstoffverbrauch von $616,69 \mathrm{ccm}$.

Die Temperatur des Thieres war

$$
38^{\circ}, 4 \mathrm{C} \text {. }
$$

Die Kohlensäureproduction pro Kilo und Stunde betrug

$$
827,03 \mathrm{ccm} \text {. }
$$

\section{Versuch II.}

Anfang des Versuches 1 Uhr 48 Min. Dauer 25 Min.

Temperatur des Thieres $38^{\circ}, 9 \mathrm{C}$.

a) Anfangsvolum auf $0^{\circ} \mathrm{C}$. und $760 \mathrm{~mm}$ reducirt . . . $=936,73 \mathrm{ccm}$

b) Endvolum anf $0^{\circ} \mathrm{C}$. und $760 \mathrm{~mm}$ reducirt . . . . =458,17 Also Sauerstoffverbrauch in $25 \mathrm{Min.} \mathrm{.} \mathrm{.} \mathrm{.} \mathrm{.} \mathrm{.} \mathrm{.} \mathrm{.}=478,56 "$ Hieraus folgt für 1 Kilo and 1 Stunde ein. Sauerstofiverbrauch von $704,76 \mathrm{ccm}$.

Die Temperatur des Thieres war

$$
38^{0}, 9 \mathrm{C} \text {. }
$$

Die Kohlensäureproduction pro Kilo und Stunde betrug

$$
644,93 \mathrm{ccm} \text {. }
$$

\section{Versuch III.}

Anfang des Versuches 2 Uhr 17 Min. Dauer 29 Min.

\section{Temperatur des Thieres $37^{\circ}, 2 \mathrm{C}$.}

$$
\text { "des Bades } 35^{\circ}, 0 \mathrm{C} \text {. }
$$

a) Anfangsvolum auf $0^{\circ} \mathrm{C}$. und $760 \mathrm{~mm}$ reducirt . . . $=784,39 \mathrm{ccm}$

b) Endvolum auf $0^{\circ}$ und $760 \mathrm{~mm}$ reducirt . . . . = $=347,05$ ” Also Sauerstoffyerbrauch in $29 \mathrm{Min}$. . . . . . . . $=437,34$,

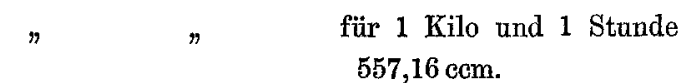

Die Temperatur des Thieres war

urr 1 Kilo und 1 Stunde

$$
37^{\circ}, 2 \mathrm{C} \text {. }
$$

Die Kohlensäureproduction pro Kilo und Stunde betrug $420,88 \mathrm{cem}$. 


\section{Versuch IV.}

Anfang des Versuches 2 Uhr 46 Min. Daner 35 Min.

Zeit . . . 2 Uhr 46 Min. $54 \quad 3$ Uhr 3 Min. $11 \quad 18$

Temperatur des

$\begin{array}{llllll}\text { Thieres . . . } & 33^{0}, 6 & 33^{\circ}, 0 & 32^{\circ}, 3 & 32^{\circ}, 0 & 31^{0}, 7\end{array}$

Temperatur des

Bades . . . $\quad 30^{\circ}, 0 \quad 29^{\circ}, 5 \quad 29^{\circ}, 0 \quad 28^{\circ}, 5 \quad 28^{\circ}, 0$

Herzschlag kräftig.

a) Anfangsvolum auf $0^{\circ}$ und $760 \mathrm{~mm}$ reducirt . . . $=970,56 \mathrm{ccm}$

b) Endvolum auf $0^{0}$ und $760 \mathrm{~mm}$ reducirt . . . . $=579,14$ ”

Also Sauerstoffverbrauch in 35 Min. . . . . . . . $=391,42$ n

" pro 1 Kilo und 1 Stunde

$413,18 \mathrm{ccm}$.

Die mittlere Temperatur des Thieres war während dieses Zeitraumes $32^{\circ}, 5 \mathrm{C}$.

Die Kohlensäureproduction pro Kilo und Stunde betrug

$440,20 \mathrm{ccm}$.

Versuch V.

Anfang des Versuches 3 Uhr 21 Min. Dauer 35 Min.

$\begin{array}{llllll}\text { Zeit . . . } & 3 \text { Uhr } 21 \text { Min. } 29 & 36 & 44 & 53\end{array}$

Temperatur des

Thieres . . . $\quad 31^{0}, 3 \quad 31^{0}, 1 \quad 30^{\circ}, 7 \quad 30^{\circ}, 4 \quad 30^{\circ}, 0$

Temperatur des

Bades . . $\quad 28^{\circ}, 0 \quad 27^{0}, 2 \quad 27^{\circ}, 0 \quad 26^{\circ}, 5 \quad 26^{\circ}, 0$

Herzschlag kräftig.

a) Anfangsvolum auf $0^{\circ}$ und $760 \mathrm{~mm}$ reducirt . . . $=779,22 \mathrm{ccm}$

b) Endvolum auf $0^{\circ}$ und $760 \mathrm{~mm}$ reducirt . . . . . = $=495,21$ ”

Also Sauerstoffverbrauch in $35 \mathrm{Min}$. . . . . . . $=284,01$ "

Hieraus folgt für 1 Kilo und 1 Stunde ein Sauerstoffverbrauch von.

$299,79 \mathrm{ccm}$.

Die mittlere Temperatur des Thieres war

$30^{\circ}, 7 \mathrm{C}$.

Die Kohlensäureproduction pro Kilo und Stunde betrug

$\mathbf{3 0 3}, 27 \mathrm{cem}$.

Versuch VI.

Anfang des Versuches 4 Uhr 17 Min. Dauer 20 Min.

$\begin{array}{llllll}\text { Zeit . . . . } & 4 \text { Uhr } 17 \text { Min. } & 19 & 24 & 29 & 34\end{array}$

Temperatur des

Thieres . .

Temperatur des

Bades

$27^{0}, 2 \quad 26^{0}, 9 \quad 26^{0}, 4 \quad 25^{0}, 8 \quad 25^{0}, 5$

$24^{0}, 0 \quad-\quad 23^{0}, 6 \quad 23^{0}, 2 \quad 23^{0}, 0$ 
Ueber Oxydation im Warmblüter bei subnormalen Temperaturen. 365

a) Anfangsvolum auf $0^{\circ}$ und $760 \mathrm{~mm}$ reducirt . . . $=975,15 \mathrm{ccm}$

b) Endvolum auf $0^{\circ}$ und $760 \mathrm{~mm}$ reducirt . . . . . $=860,48$ " Also Sauerstoffverbrauch in $20 \mathrm{Min}$. . . . . . . $=114,67 "$

für 1 Kilo und 1 Stunde

$211,83 \mathrm{ccm}$.

Die mittlere Temperatur des Thieres war $26^{\circ}, 3 \mathrm{C}$.

Die Kohlensäureproduction pro Kilo und Stande betrug $260,43 \mathrm{ccm}$.

\section{Versuch VII.}

Anfang des Versuches 4 Uhr 37 Min. Dauer 25 Min.

Zeit . . . 4 Uhr 38 Min. $43 \quad 48 \quad 59 \quad 5$ Uhr 3 Min.

Temperatur des

Thieres . . . $\quad 25^{0}, 5 \quad 25^{0}, 2 \quad 25^{0}, 0 \quad 24^{0}, 8 \quad 24^{0}, 5$

Temperatur des

Bades . . . $\quad 23^{0}, 0 \quad 23^{0}, 0 \quad 22^{0}, 8 \quad 22^{0}, 5 \quad-$

Herzschlag nicht fühlbar.

a) Anfangsvolum auf $0^{\circ}$ und $760 \mathrm{~mm}$ reducirt . . . . = $=754,71 \mathrm{ccm}$

b) Endvolum auf $0^{\circ}$ and $760 \mathrm{~mm}$ reducirt . . . . . $=567,65$,

Also Sauerstoffverbrauch in $25 \mathrm{Min}$. . . . . . . . $=187,06$ "

" $\quad$ für 1 Kilo und 1 Stunde

$276,44 \mathrm{ccm}$.

Die mittlere Temperatur war

$25^{\circ}, 0 \mathrm{C}$.

Die Kohlensäureproduction pro Kilo und Stunde betrug

$178,58 \mathrm{ccm}$.

Sectionsbefund: Pulsirendes Herz. Blut hellroth. Lunge Iufthaltig. Keine Athembewegungen.

Tabelle von Serie I.

\begin{tabular}{|c|c|c|c|c|c|}
\hline $\begin{array}{c}\text { Nummer } \\
\text { des Ver- } \\
\text { suches. }\end{array}$ & $\begin{array}{l}\text { Sauerstoffver- } \\
\text { brauch pro } \\
1 \text { Kilo und } \\
1 \text { Stunde in ccm } \\
\text { auf } 0^{\circ} \text { und } \\
0,76 \mathrm{M} \text {. reducirt. }\end{array}$ & $\begin{array}{c}\text { Tempera- } \\
\text { tur des } \\
\text { Thieres, } \\
\text { eventuell } \\
\text { mittlere. }\end{array}$ & $\begin{array}{c}\text { Angabe der } \\
\text { Grenzen, inner- } \\
\text { halb welcher } \\
\text { die Temperatur } \\
\text { des Thieres } \\
\text { schwankte. }\end{array}$ & \begin{tabular}{|} 
Dauer des \\
einzelnen \\
Ver- \\
suches in \\
Minuten.
\end{tabular} & $\begin{array}{l}\text { Kohlensäure- } \\
\text { ausscheidung } \\
\text { pro Kilo und } \\
\text { Stunde in ccm } \\
\text { auf } 0^{\circ} \text { und } \\
0,76 \mathrm{M} \text {. reducirt. }\end{array}$ \\
\hline 1. & 616,69 & $38,4^{\circ} \mathrm{C}$ & - & 20 & 827,03 \\
\hline 2. & & 38,9 & - & 25 & 644,93 \\
\hline 3. & 55 & $37,2 "$ & - & 29 & 420,88 \\
\hline 0. & 413,18 & $32,5 "$ & $\mid \begin{array}{c}\text { Sinkt von } 33^{0}, 6 \\
\text { auf } \mathbf{3 1}^{0}, \mathbf{7}\end{array}$ & 35 & 440,20 \\
\hline 5. & 299,79 & 30,7 & $\mid \begin{array}{c}\text { Sinkt von } 31^{\circ}, 3 \\
\text { auf } 30^{\circ}, 0 .\end{array}$ & 35 & 303,27 \\
\hline 6. & 211,83 & $26,3 n$ & Sinkt von $27^{\circ}, 2$ & 20 & 260,43 \\
\hline 7. & 276,44 & 25,0 & $\begin{array}{l}\text { Sinkt von } 25^{\circ}, 5 \\
\text { auf } 24^{0}, 5 \text {. }\end{array}$ & 25 & 178,58 \\
\hline
\end{tabular}




\section{Serie II (28. Nov.)}

Kaninchen von $1830 \mathrm{gr}$ curarisirt.

\section{Versuch I.}

Anfang des Versuches $12 \mathrm{Uhr} 16 \mathrm{Min}$. Dauer $20 \mathrm{Min}$.

$\begin{array}{lllll}\text { Zeit . . . } & 12 \text { Uhr } 16 \text { Min. } 26 & 31 & 36\end{array}$

Temperatur des

Thieres. . .

Temperatur des

$\begin{array}{lllll}\text { Bades . . . } & 37^{0}, 8 & 37^{\circ}, 5 & 37^{0}, 0 & 37^{0}, 5\end{array}$

a) Anfangsvolum auf $0^{0}$ und $760 \mathrm{~mm}$ reducirt . . . $=606,47 \mathrm{ccm}$

b) Endvolum auf $0^{\circ}$ und $760 \mathrm{~mm}$ reducirt . . . . . $=261,65$ " Also Samerstoffverbrauch in 20 Minuten . . . . . $=344,82$ " Hieraus Sauerstoffverbrauch für 1 Kilo und 1 Stunde

$565,28 \mathrm{~cm}$.

Die mittlere Temperatur des Thieres war $37^{\circ}, 5 \mathrm{C}$.

Die Kohlensäureproduction pro Kilo und Stunde betrug $405,70 \mathrm{ccm}$.

Versuch II.

Anfang des Versuches 12 Uhr 36 Min. Dauer 20 Min.

Zeit . . . 12 Uhr 41 Min. $46^{\circ} \quad 51 \quad 56$

Temperatur des

$\begin{array}{lllll}\text { Thieres . . . } & 37^{0}, 7 & 37^{0}, 7 & 37^{0}, 7 & 37^{0}, 7\end{array}$

Temperatur des

Bades . . $\quad 36^{\circ}, 0 \quad 36^{0}, 0 \quad 35^{0}, 0 \quad 35^{\circ}, 0$

a) Anfangsvolum auf $0^{\circ}$ und $760 \mathrm{~mm}$ reducirt . . . $=952,80 \mathrm{~cm}$

b) Endvolum auf $0^{\circ}$ und $760 \mathrm{~mm}$ reducirt . . . . $=579,48$ " Also Sauerstoffverbrauch in $20 \mathrm{Min}$. . . . . . . $=373,32 "$

Hieraus Sauerstoffverbrauch für 1 Kilo und 1 Stunde

$612,00 \mathrm{ccm}$.

Die mittlere Temperatur des Thieres war $37^{0}, 7 \mathrm{C}$.

Die Kohlensäureproduction pro Kilo und Stunde betrug $773,64 \mathrm{cem}$.

Jetzt wurde das Thier durch Uebergiessen mit kaltem Wasser abgekühlt und in ein kälteres Bad gebracht.

Versuch III.

Anfang des Versuches 1 Uhr 20 Min. Dauer 20 Min.

Zeit . . . 1 Uhr 20 Min. 25 $30 \quad 35 \quad 40$

Temperatur des

Thieres . .

Temperatur des

Bades . .

$33^{0}, 0 \quad 32^{0}, 5 \quad 32^{0}, 1 \quad 32^{0}, 0 \quad 31^{0}, 8$

$30^{\circ}, 0 \quad 30^{\circ}, 0 \quad 30^{\circ}, 0 \quad 29^{\circ}, 7 \quad 29^{\circ}, 3$


a) Anfangsvolum auf $0^{\circ}$ und $760 \mathrm{~mm}$ reducirt . . . $=718,59 \mathrm{~cm}$

b) Endvolum auf $0^{\circ}$ und $760 \mathrm{~mm}$ reducirt . . . . . $=415,69$ " Also Sauerstoffverbrauch in 20 Min. . . . . . . . $=302,90$ " Hieraus Sauerstoffiverbrauch für 1 Kilo und 1 Stunde $496,56 \mathrm{ccm}$.

Die mittlere Temperatur des Thieres war $32^{\circ}, 3 \mathrm{C}$.

Die Kohlensänreproduction pro Kilo und Stunde betrug $520,41 \mathrm{ccm}$.

\section{Versuch IV.}

Anfang des Versuehes $1 \mathrm{Uhr} 40 \mathrm{Min}$. Dauer $20 \mathrm{Min}$.

Zeit . . . 1 Uhr $45 \mathrm{Min} . \quad 50 \quad 55 \mathrm{Uhr}$

Temperatur des

Thieres . . .

Temperatur des

Bades . . .

$$
31^{0}, 8 \quad 31^{0}, 6 \quad 31^{0}, 6 \quad 31^{0}, 5
$$

$29^{\circ}, 0 \quad 29^{\circ}, 0 \quad 28^{\circ}, 7 \quad 28^{\circ}, 0$

a) Anfangsvolum auf $0^{\circ}$ und $760 \mathrm{~mm}$ reducirt . . . $=870,77 \mathrm{~cm}$

b) Endvolum auf $0^{\circ}$ und $760 \mathrm{~mm}$ reducirt . . . = $=559,64$ '” Also Sauerstoffverbrauch in $20 \mathrm{Min}$. . . . . . . $=311,13 "$ Hieraus Sauerstoffverbrauch für 1 Kilo und 1 Stunde

$$
510,05 \mathrm{ccm} \text {. }
$$

Die mittlere Temperatur des Thieres war

$$
31^{0}, 6 \mathrm{C} \text {. }
$$

Die Kohlensäureproduction pro Kilo und Stunde betrug

$$
460,67 \mathrm{ccm} \text {. }
$$

Nochmalige Abkühlung des Thieres und des Bades.

\section{Versuch V.}

Anfang des Versuches $2 \mathrm{Uhr} 35 \mathrm{Min}$. Dauer $20 \mathrm{Min}$.

Zeit . . . . 2 Uhr 35 Min. $40 \quad 45 \quad 50$

\section{Temperatur des}

Thieres . .

Temperatur des

$$
26^{0}, 7 \quad 26^{0}, 4 \quad \cdot \quad 26^{0}, 0 . \quad 25^{0}, 8
$$

Bades . . .

$$
24^{0}, 8 \quad 24^{0}, 5 \quad 24^{0}, 4 \quad 24^{\circ}, 0
$$

a) Anfangsvolum auf $0^{\circ}$ und $760 \mathrm{~mm}$ reducirt . . . $=657,30 \mathrm{ccm}$

b) Endvolum auf $0^{\circ}$ und $760 \mathrm{~mm}$ reducirt . . . . . $=559,00 "$ Also Sanerstofiverbrauch in 20 Min. . . . . . . . $=98,30 \%$ Hieraus Sauerstoffverbrauch für 1 Kilo und 1 Stunde

$$
161,15 \mathrm{ccm} \text {. }
$$

Die mittlere Temperatur des Thieres war

$$
26^{\circ}, 2 \mathrm{C} \text {. }
$$

Die Kohlensäureproduction pro Kilo und Stunde betrug

\section{$211,18 \mathrm{ccm}$.}




\section{Versuch VI.}

Anfang des Versuches 2 Uhr 55 Min. Dauer 20 Min.

Zeit . . . $\quad 2$ Uhr 55 Min. 3 Uhr $10 \quad 15$

Temperatur des

Thieres . . .

Temperatar des

Bades . . .

$$
25^{0}, 7
$$

$25^{0}, 6$

$25^{0}, 5$

$25^{0}, 5$

a) Anfangsvolum auf $0^{\circ}$ und $760 \mathrm{~mm}$ reducirt . . . . $=835,13 \mathrm{ccm}$

b) Endvolum auf $0^{0}$ und $760 \mathrm{~mm}$ reducirt . . . . . = 723,27 "

Also Sauerstoffverbrauch in $20 \mathrm{Min}$. . . . . . . . = $=111,86 "$

Hieraus Sauerstoffverbrauch für 1 Kilo und 1 Stunde

$183,38 \mathrm{ccm}$.

Die mittlere Temperatur des Thieres war

$$
25^{0}, 6 \mathrm{C} \text {. }
$$

Die Kohlensäureproduction pro Kilo und Stunde betrug

\section{$178,73 \mathrm{ecm}$.}

Sectionsbefund: Herzschlag kräftig, Lungen lufthaltig, hell. Blut hellroth, Venen hellroth, besonders Leber. Kräftige Herzcontractionen. Peristaltische Bewegungen des Darmes.

\begin{tabular}{|c|c|c|c|c|c|}
\hline $\begin{array}{l}\text { Nummer } \\
\text { des Ver- } \\
\text { suches. }\end{array}$ & $\begin{array}{c}\text { Sauerstoffver- } \\
\text { brauch pro } \\
1 \text { Kilo und } \\
1 \text { Stunde in cem } \\
\text { auf } 0^{\circ} \text { und } \\
0,76 \mathrm{M} \text {. reducirt. }\end{array}$ & $\begin{array}{l}\text { Tempera- } \\
\text { tur des } \\
\text { Thieres, } \\
\text { eventuelI } \\
\text { mittlere. }\end{array}$ & \begin{tabular}{|c} 
Angabe der \\
Grenzen inner- \\
halb welcher \\
die Temperatur \\
des Thieres \\
schwankte.
\end{tabular} & $\begin{array}{l}\text { Dauer des } \\
\text { einzelnen } \\
\text { Ver- } \\
\text { suches in } \\
\text { Minuten. }\end{array}$ & $\begin{array}{l}\text { Kohlensäure } \\
\text { ausscheidung } \\
\text { pro Kilo und } \\
\text { Stunde in cem } \\
\text { auf } 0^{\circ} \text { und } \\
0,76 \mathrm{M} \text {. reducirt. }\end{array}$ \\
\hline 1. & 565,28 & $37,5^{\circ} \mathrm{C}$ & $\begin{array}{c}\text { Fiel von } 37^{\circ}, 6 \\
\text { auf } 37^{\circ}, 4 \text { und } \\
\text { stieg wieder } \\
\text { auf } 37^{\circ}, 6 .\end{array}$ & 20 & 405,70 \\
\hline 2. & 612,00 & $37,7 \%$ & Constant. & 20 & 773,64 \\
\hline 3 & 496,56 & & $\begin{array}{c}\text { Fiel von } 33^{\circ}, 0 \\
\text { anf } 31^{0}, 8 \text {. }\end{array}$ & 20 & 520,41 \\
\hline 4. & 510,05 & 31,6 & $\begin{array}{c}\text { Fiel von } 31^{\circ}, 8 \\
\text { auf } 31^{0}, 5 .\end{array}$ & 20 & 460,67 \\
\hline 5. & 161,15 & 26,2 & $\begin{array}{c}\text { Fiel von } 26^{\circ}, 7 \\
\text { auf } 25^{\circ}, 8\end{array}$ & 20 & 211,18 \\
\hline 6. & 183,38 & 25,6 & $\begin{array}{l}\text { Fiel von } 25^{0}, 7 \\
\text { auf } 25^{0}, 5 \text {. }\end{array}$ & 20 & 178,73 \\
\hline
\end{tabular}

Tabelle von Serie II. 
Ueber Oxydation im Warmblüter bei subnormalen Temperaturen. 369

\section{Serie III (7. Dee.)}

Kaninchen von 2007 gr curarisirt.

\section{Versuch I.}

Anfang des Versuches $10 \mathrm{Uhr} 10 \mathrm{Min}$. Daner $20 \mathrm{Min}$.

Zeit . . . $10 \mathrm{Uhr} 10 \mathrm{Min} .15 \quad 20 \quad 25 \quad 30$

Temperatur des

$\begin{array}{llllll}\text { Thieres . . . } & 37^{0}, 3 & 37^{0}, 3 & 37^{0}, 4 & 37^{0}, 5 & 37^{0}, 5\end{array}$

Temperatur des

Bades . . $\quad 37^{0}, 0 \quad 36^{0}, 5 \quad 36^{0}, 0 \quad 35^{0}, 5 \quad 35^{0}, 0$

a) Anfangsvolum auf $0^{\circ}$ und $760 \mathrm{~mm}$ reducirt . . . $=760,91 \mathrm{ccm}$

b) Endvolum auf $0^{\circ}$ und $760 \mathrm{~mm}$ reducirt . . . . . $=444,58$ "

Also Sauerstoffverbrauch in 20 Min. . . . . . . . $=316,33$ "

Hieraus Sauerstoffverbrauch für 1 Kilo und 1 Stunde

$472,84 \mathrm{ccm}$.

Die mittlere Temperatur des Thieres war

$37^{\circ}, 4 \mathrm{C}$.

Die Kohlensäureproduction pro Kilo und Stunde betrug

$511,45 \mathrm{ccm}$.

Versuch II.

Anfang des Versuches 10 Uhr 30 Min. Dauer 20 Min.

Zeit . . . 10 Uhr 30 Min. $35 \quad 40 \quad 45 \quad 50$

Temperatur des

Thieres . . . $\quad 37^{\circ}, 5 \quad 37^{0}, 4 \quad 37^{0}, 4 \quad-\quad 37^{0}, 2$

Temperatur des

Bades . . . $\quad 35^{0}, 0 \quad 34^{0}, 5 \quad 34^{0}, 0 \quad-\quad 34^{0}, 0$

a) Anfangsvolum auf $0^{\circ}$ und $760 \mathrm{~mm}$ reducirt . . . $=959,56 \mathrm{ccm}$

b) Endvolum auf $0^{\prime \prime}$ und $760 \mathrm{~mm}$ reducirt . . . . . = $=656,72$ "

Also Sauerstoffverbrauch in 20 Min. . . . . . . $=302,84$,

Hieraus Sauerstoffverbrauch für 1 Kilo und 1 Stunde

$452,67 \mathrm{~cm}$.

Die mittlere Temperatur des Thieres war

$$
37^{\circ}, 4 \mathrm{C} \text {. }
$$

Die Kohlensäureproduction pro Kilo und Stunde betrug

\section{$491,46 \mathrm{ccm}$.}

Versuch III.

Vor diesem Versuche wurden Thier und Bad abgekühlt.

Anfang des Versuches $11 \mathrm{Uhr} 20$ Min. Dauer $20 \mathrm{Min}$.

Zeit . . . 11 Uhr 20 Min. 25 $\quad 30 \quad 35 \quad 40$

Temperatur des

$\begin{array}{llllll}\text { Thieres . . . } & 32^{0}, 6 & 31^{0}, 7 & 31^{0}, 1 & 30^{0}, 8 & 30^{\circ}, 7\end{array}$

Temperatur des

Bades . . $\quad 29^{\circ}, 0 \quad 28^{\circ}, 0 \quad 28^{\circ}, 0 \quad 28^{\circ}, 0 \quad 27^{\circ}, 0$ 
a) Anfangsvolum auf $0^{\circ}$ und $760 \mathrm{~mm}$ reducirt . . . $=775,73 \mathrm{ccm}$

b) Endvolum auf $0^{\circ}$ und $760 \mathrm{~mm}$ reducirt . . . . . $=522,90$ " Also Sauerstoffverbrauch in $20 \mathrm{Min}$. . . . . . . $=252,83$ " Hieraus Sauerstoffverbrauch für 1 Kilo und 1 Stunde $377,92 \mathrm{ccm}$.

Die mittlere Temperatur des Thieres war

$$
31^{\circ}, 4 \mathrm{C} \text {. }
$$

Die Kohlensäureproduction pro Kilo und Stunde betrug $354,62 \mathrm{ccm}$.

\section{Versuch IV.}

Anfang des Versuches $11 \mathrm{Uhr} 40 \mathrm{Min}$. Dauer $20 \mathrm{Min}$.

Zeit . . . 11 Uhr 40 Min. $45 \quad 50 \quad 55 \quad 12$ Uhr

\section{Temperatur des}

Thieres. . .

Temperatur des

Bades . . .

$\begin{array}{rrrrr}30^{\circ}, 7 & 30^{\circ}, 8 & 30^{\circ}, 7 & 30^{\circ}, 3 & 30^{\circ}, 2 \\ 27^{\circ}, 0 & 27^{\circ}, 0 & 27^{\circ}, 0 & 27^{\circ}, 0 & 26^{\circ}, 5\end{array}$

a) Anfangsvolum anf $0^{\circ}$ and $760 \mathrm{~mm}$ reducirt . . . $=946,66 \mathrm{ccm}$

b) Endvolum auf $0^{\circ}$ und $760 \mathrm{~mm}$ reducirt . . . . . = 714,75 "

Also Sauerstoffverbrauch in $20 \mathrm{Min}$. . . . . . . $=231,91$ "

Hieraus Sauerstoffverbrauch für 1 Kilo und 1 Stunde

$346,65 \mathrm{ccm}$.

Die mittlere Temperatur des Thieres war

$30^{\circ}, 5 \mathrm{C}$.

Die Kohlensäureproduction pro Kilo und Stunde betrug

$296,34 \mathrm{ccm}$.

Versuch V.

Anfang des Versuches $12 \mathrm{Uhr}$. Dauer $25 \mathrm{Min}$.

\begin{tabular}{|c|c|c|c|c|c|}
\hline Zeit . . . . & $12 \mathrm{Uhr}$ & 5 & 10 & 15 & 20 \\
\hline $\begin{array}{c}\text { Temperatur des } \\
\text { Thieres. . . }\end{array}$ & $30^{\circ}, 2$ & $30^{\circ}, 0$ & $29^{0}, 8$ & $29^{0}, 6$ & $29^{0}, 3$ \\
\hline $\begin{array}{c}\text { Temperatur des } \\
\text { Bades }\end{array}$ & $26^{0}, 5$ & $26^{\circ}, 0$ & $26^{0}, 0$ & $25^{0}, 5$ & $25^{0}, 0$ \\
\hline
\end{tabular}

Durch ein Versehen wurde aus den Respirationsventilen die Kalilange entleert am Ende des Versuches, noch bevor die Ablesung am Spirometer I gemacht war. Deshalb ergab dieser Versuch keine Resultate.

\section{Versuch VI.}

Anfang des Versuches 12 Uhr 25 Min. Dauer 20 Min.

\begin{tabular}{|c|c|c|c|c|c|}
\hline Zeit . . . . & 12 Uhr 25 Min. & 30 & 35 & 40 & 45 \\
\hline $\begin{array}{c}\text { Temperatur des } \\
\text { Thieres . . }\end{array}$ & $29^{\circ}, 2$ & $29^{\circ}, 1$ & $28^{\circ}, 8$ & $28^{0}, 7$ & $28^{\circ}, 2$ \\
\hline $\begin{array}{c}\text { Temperatur des } \\
\text { Bades } . . .\end{array}$ & $25^{\circ}, 0$ & $25^{0}, 0$ & $25^{0}, 0$ & $24^{\circ}, 5$ & $24^{0}, 0$ \\
\hline
\end{tabular}

a) Anfangsvolum auf $0^{\circ}$ und $760 \mathrm{~mm}$ reducirt . . . $=942,94 \mathrm{ccm}$

b) Endvolum auf $0^{\circ}$ und $760 \mathrm{~mm}$ reducirt . . . . . $=807,41$ "

Also Sauerstoffverbrauch in $20 \mathrm{Min}$. . . . . . . $=135,53$, 
Ueber Oxydation im Warmblüter bei subnormalen Temperaturen. 371

Hieraus Sauerstoffverbrauch für 1 Kilo und 1 Stunde

$$
\text { - } 202,58 \mathrm{ccm} \text {. }
$$

Die mittlere Temperatur des Thieres war

$$
28^{0}, 8 \mathrm{C} \text {. }
$$

Die Kohlensäureproduction pro Kilo und Stunde betrug $366,38 \mathrm{cem}$.

\section{Versuch VII.}

Anfang des Versuches 1 Uhr. Dauer 20 Min.

$\begin{array}{llllllll}\text { Zeit . . . . } & 1 \text { Uhr } & 5 & 10 & 15 & 20\end{array}$

Temperatur des

Thieres . .

Temperatur des

Bades . .

$$
27^{0}, 5 \quad 27^{0}, 2 \quad 27^{\circ}, 0 \quad 26^{0}, 7 \quad 26^{0}, 5
$$

$23^{0}, 0 \quad 23^{0}, 0 \quad 22^{0}, 8 \quad 23^{0}, 0 \quad 22^{0}, 5$

Herzschlag nicht fühlbar.

a) Anfangsvolum auf $0^{\circ}$ und $760 \mathrm{~mm}$ reducirt . . . $=749,88 \mathrm{ccm}$

b) Endvolum auf $0^{\circ}$ und $760 \mathrm{~mm}$ reducirt . . . . . $=621,30$ ” Also Sauerstoffverbrauch in $20 \mathrm{Min}$. . . . . . . . $=128,58$ " Hieraus Sauerstoffverbrauch für 1 Kilo und 1 Stunde

$192,19 \mathrm{ccm}$.

Die mittlere Temperatur des Thieres war

$$
27^{\circ}, 0 \mathrm{C} \text {. }
$$

Die Kohlensäureproduction pro Kilo und Stunde betrug

$$
101,02 \mathrm{ccm} \text {. }
$$

\begin{tabular}{|c|c|c|c|c|c|}
\hline $\begin{array}{l}\text { Nummer } \\
\text { des Ver- } \\
\text { suches. }\end{array}$ & $\begin{array}{l}\text { Sauerstoffver- } \\
\text { brauch pro } \\
1 \text { Kilo und } \\
1 \text { Stunde in ccm } \\
\text { auf } 0^{\circ} \text { und } \\
0,76 \mathrm{M} \text {. reducirt. }\end{array}$ & $\begin{array}{l}\text { Tempera- } \\
\text { tur des } \\
\text { Thieres, } \\
\text { eventuell } \\
\text { mittlere. }\end{array}$ & \begin{tabular}{|c} 
Angabe der \\
Grenzen inner- \\
halb welcher \\
die Temperatur \\
dee Thieres \\
schwankte.
\end{tabular} & $\mid \begin{array}{c}\text { Dauer des } \\
\text { einzelnen } \\
\text { Ver- } \\
\text { suches in } \\
\text { Minuten. }\end{array}$ & $\begin{array}{l}\text { Kohlensäure- } \\
\text { ausscheidung } \\
\text { pro Kilo und } \\
\text { Stunde in cem } \\
\text { auf } 0^{\circ} \text { und } \\
0,76 \mathrm{M} \text {. xeducirt. }\end{array}$ \\
\hline 1. & 472,84 & $37,4^{\circ} \mathrm{C}$ & $\begin{array}{c}\text { Stieg ron } 37^{\circ}, 3 \\
\text { auf } 37^{0}, 5 .\end{array}$ & 20 & 511,45 \\
\hline 2. & 452,67 & 37,4, & $\begin{array}{l}\text { Fiel von } 37^{\circ}, 5 \\
\text { auf } 37^{\circ}, 2\end{array}$ & 20 & 491,46 \\
\hline 3. & 377,92 & $31,4 n$ & $\begin{array}{c}\text { Fiel von } 32^{\circ}, 6 \\
\text { auf } 30^{\circ}, 7 .\end{array}$ & 20 & 354,62 \\
\hline 4. & 346,65 & 30,5 & $\begin{array}{l}\text { Stieg von } 30^{\circ}, 7 \\
\text { auf } 30^{\circ}, 8 \text { und } \\
\text { fiel dann auf } \\
30^{\circ}, 2\end{array}$ & 20 & 296,34 \\
\hline $\begin{array}{l}5 . \\
6 .\end{array}$ & $\begin{array}{l}\text { verunglückt } \\
202,58\end{array}$ & $2 \overline{8} \overline{8}$ & Fiel von $29^{\circ}, 2$ & $\overline{20}$ & $\overline{366,38}$ \\
\hline 7. & 192,19 & 27,0 & $\begin{array}{l}\text { auf } 28^{0}, 2 . \\
\text { Fiel von } 27^{0}, 5 \\
\text { auf } 26^{0}, 5 .\end{array}$ & 20 & 101,02 \\
\hline
\end{tabular}

Sectionsbefund: Unregelmässig pulsirendes Herz. Blut ziemlich hellroth.

Tabelle von Serie III. 


\section{Serie IV (30. Dec.)}

Kaninchen von $1471 \mathrm{gr}$ curarisirt.

\section{Versuch I.}

Anfang des Versuches 1 Uhr 15 Min. Dauer 20 Min.

$\begin{array}{lcc}\text { Zeit } . \cdot . \cdot \cdot \text { Uhr 15 Min. } & 1 \text { Uhr 35 Min. } \\ \text { Temperatur des Thieres } & 38^{\circ}, 3 & 38^{\circ}, 3 \\ \text { Temperatur des Bades } & 36^{\circ}, 0 & 34^{\circ}, 5\end{array}$

a) Anfangsvolum auf $0^{\circ}$ und $760 \mathrm{~mm}$ reducirt . . . $=759,91 \mathrm{ccm}$

b) Endvolum auf $0^{\circ}$ und $760 \mathrm{~mm}$ reducirt . . . . . $=511,74$ " Also Sauerstoffverbrauch in $20 \mathrm{Min}$. . . . . . . . $=248,17$, Hieraus Sauerstoffverbrauch für 1 Kilo und 1 Stunde $506,12 \mathrm{ccm}$

Die mittlere Temperatur des Thieres war $38^{\circ}, 3$ C.

Die Kohlensäureproduction pro Kilo und Stunde betrug $487,87 \mathrm{ccm}$.

\section{Versuch II.}

Anfang des Versuches $1 \mathrm{Uhr} 35 \mathrm{Min}$. Dauer $20 \mathrm{Min}$.

$\begin{array}{lcc}\text { Zeit } . . . \cdot . & 1 \mathrm{Uhr} 35 \mathrm{Min} . & 1 \mathrm{Uhr} 55 \mathrm{Min} . \\ \text { Temperatur des Thieres } & 38^{0}, 3 & 38^{\circ}, 3 \\ \text { Temperatur des Bades } & 34^{\circ}, 5 & 33^{0}, 0\end{array}$

a) Anfangsvolum auf $0^{\circ}$ und $760 \mathrm{~mm}$ reducirt . . . $=931,41 \mathrm{ccm}$

b) Endvolum auf $0^{\circ}$ und $760 \mathrm{~mm}$ reducirt . . . . . $=669,28$ n Also Sauerstoffverbrauch in $20 \mathrm{Min.} \mathrm{.} \mathrm{.} \mathrm{.} \mathrm{.} \mathrm{.} \mathrm{.} \mathrm{.}=262,13$ " Hieraus Sauerstoffverbrauch für 1 Kilo und 1 Stunde $534,59 \mathrm{ccm}$.

Die mittlere Temperatur des Thieres war $38^{\circ}, 3 \mathrm{C}$.

Die Kohlensäureproduction pro Kilo and Stunde betrug $487,72 \mathrm{ecm}$.

\section{Versuch III.}

Vor diesem Versuche wurde das Thier durch Uebergiessen mit kaltem Wasser abgekühlt and in ein kälteres Bad gebracht; unterdessen wurde die künstliche Respiration, wie bei allen Unterbrechungen der Versuchsreihe, mittelst eines Blasebalges bewirkt.

Anfang des Versuches 2 Uhr 50 Min. Dauer 20 Min. 
Ueber Oxydation im Warmblüter bei subnormalen Temperaturen. 373 Zeit . . 2 Uhr 50 Min. 3 Uhr. $5 \quad 10$

Temperatur des

Thieres . .

Temperatur des

Bates . .

$$
28,0 \quad 27,7 \quad 27,4 \quad 27^{\circ}, 2
$$

$24,0 \quad 23,0 \quad 23,0 \quad 23^{\circ}, 0$

a) Anfangsvolum auf $0^{\circ}$ und $760 \mathrm{~mm}$ reducirt . . . $=741,96 \mathrm{~cm}$.

b) Endvolum auf $0^{\circ}$ und $760 \mathrm{~mm}$ reducirt . . . . . $=626,69$ ” Also Sauerstoffverbrauch in $20 \mathrm{Min}$. . . . . . . $=115,27 "$ Hieraus Sauerstoffverbrauch für 1 Kilo und 1 Stunde

$235,08 \mathrm{ccm}$.

Die mittlere Temperatur des Thieres war

$$
27^{0}, 6 \mathrm{C} \text {. }
$$

Die Kohlensäureproduction pro Kilo und Stunde betrug

$\mathbf{2 3 4}, 82 \mathrm{ccm}$.

\section{Versuch IV.}

Anfang des Versuches 3 Uhr 10 Min. Dauer $20 \mathrm{Min}$.

Zeit . . . $\quad 3$ Uhr 10 Min. $\quad 20 \quad 25 \quad 30$

Temperatur des

Thieres. . .

Temperatur des

Bades . .

27,2

$26,7 \quad 26,3 \quad 26^{\circ}, 2$

23,0

$22,5 \quad 22,0 \quad 22^{\prime \prime}, 0$

a) Anfangsvolum auf $0^{\circ}$ und $760 \mathrm{~mm}$ reducirt . . . . $=949,61 \mathrm{ccm}$

b) Endvolum auf $0^{\circ}$ und $760 \mathrm{~mm}$ reducirt . . . . $=863,50$,

Also Sauerstoffverbrauch in $20 \mathrm{Min}$. . . . . . . . $=86,11$ "

Hieraus Sanerstoffrerbrauch fïr 1 Kilo und 1 Stunde

$175,61 \mathrm{ccm}$.

Die mittlere Temperatur des Thieres war

$26^{0}, 6 \mathrm{C}$.

Die Kohlensäureproduction pro Kilo and Stunde betrug

$184,37 \mathrm{ccm}$.

\section{Versuch V.}

Vor diesem Versuche wurde die Temperatur des Thieres durch Uebergiessen mit wärmerem Wasser wieder erhöht und das Thier in ein wärmeres Bad gebracht.

Anfang des Versuches 4 Uhr 50 Min. Dauer 25 Min.

Zeit . . . 4 Uhr 50 Min. $55 \quad 5$ Uhr $5 \quad 10 \quad 15$

Temperatur des

$\begin{array}{lllllll}\text { Thieres . . . } & 36,0 & 36,3 & 36,3 & \mathbf{3 6 , 3} & \mathbf{3 6 , 5} & \mathbf{3 6 0}, 7\end{array}$

Temperatur des

Bades . . $\quad 39,0 \quad 39,0 \quad 39,0 \quad 38,0 \quad 38,0 \quad 37^{\circ}, 5$

a) Anfangsvolum auf $0^{\circ}$ und $760 \mathrm{~mm}$ reducirt . . . $=767,29 \mathrm{~cm}$

b) Endvolum anf $0^{\circ}$ und $760 \mathrm{~mm}$ reducirt . . . . . $=462,45 \%$ 
Also Sauerstoffverbrauch in 25 Min. . . . . . . $=304,84 \mathrm{ccm}$.

Hieraus Sauerstoffverbrauch für 1 Kilo und 1 Strnde

$$
497,36 \mathrm{ccm} \text {. }
$$

Die mittlere Temperatur des Thieres war

$36^{\circ}, 4 \mathrm{C}$.

Die Bestimmung der Kohlensäureproduction war wegen Zerbrechens der die Kalilauge enthaltenden Flasche unmöglich.

\section{Versuch VI.}

Anfang des Versuches 5 Uhr 20 Min. Dauer 30 Min.

Zeit . . . 5 Uhr 20 Min. $\quad 30 \quad 40 \quad 40$

Temperatur des

Thieres. . .

$$
36,7
$$

36,7

36,6

$36^{0}, 8$

Temperatur des

Bades . . $\quad 37,0 \quad 38,0 \quad 37,5 \quad 36^{\circ}, 0$

a) Anfangsvolum auf $0^{\circ}$ und $760 \mathrm{~mm}$ reducirt . . . $=972,73 \mathrm{ccm}$

b) Endvolum auf $0^{\circ}$ und $760 \mathrm{~mm}$ reducirt . . . . . = $=610,79$ " Also Sauerstoffverbrauch in $30 \mathrm{Min}$. . . . . . . . $=361,94$ " Hieraus Sauerstoffverbrauch für 1 Kilo und 1 Stunde

$$
492,10 \mathrm{ccm} \text {. }
$$

Die mittlere Temperatur des Thieres war

$$
36^{\circ}, 7 \text { C. }
$$

Die Kohlensäureproduction pro Kilo und Stunde betrug

$$
497,64 \mathrm{cem} \text {. }
$$

\begin{tabular}{|c|c|c|c|c|c|}
\hline $\begin{array}{c}\text { Nummer } \\
\text { des Ver- } \\
\text { suches. }\end{array}$ & $\begin{array}{l}\text { Sauerstoffver- } \\
\text { brauch pro } \\
1 \text { Kilo and } \\
1 \text { Stunde in ecm } \\
\text { auf } 0^{\circ} \text { und } \\
0,76 \mathrm{M} \text {. reducirt. }\end{array}$ & $\begin{array}{l}\text { Tempera- } \\
\text { tur des } \\
\text { Thieres, } \\
\text { eventuell } \\
\text { mittlere. }\end{array}$ & $\begin{array}{c}\text { Angabe der } \\
\text { Grenzen, inner- } \\
\text { halb welcher } \\
\text { die Temperatur } \\
\text { des Thieres } \\
\text { schwankte. }\end{array}$ & $\begin{array}{c}\text { Dauer des } \\
\text { einzelnen } \\
\text { Ver- } \\
\text { suches in } \\
\text { Minuten. }\end{array}$ & $\begin{array}{l}\text { Kohlensäure- } \\
\text { ausscheidung } \\
\text { pro Kilo und } \\
\text { Stunde in ccm } \\
\text { auf } 0^{\circ} \text { und } \\
0,76 \mathrm{M} \text {. reducirt. }\end{array}$ \\
\hline 1. & & $38,3^{\circ} \mathrm{C}$. & Cor & 20 & 487,87 \\
\hline 2. & & & Constant. & & 48 \\
\hline 3. & 2 & 27,6 & $\begin{array}{c}\text { Fiel von } 28^{\circ}, 0 \\
\text { auf } 27^{\circ}, 2\end{array}$ & & \\
\hline 4. & 175,61 & $26,6 "$ & $\begin{array}{l}\text { Fiel von } 27^{\circ}, 2 \\
\text { auf } 26^{\circ}, 2 .\end{array}$ & 20 & 184,37 \\
\hline 5. & 497,36 & $36,4 ”$ & $\begin{array}{l}\text { Stieg von } 36^{\circ}, 0 \\
\text { auf } 36^{0}, 7\end{array}$ & 25 & verunglückt. \\
\hline 6. & 492,10 & $36,7 \#$ & $\mid \begin{array}{r}\text { Fiel von } 36^{\circ}, 7 \\
\text { auf } 36^{0}, 6 \text { und } \\
\text { stieg auf } 36^{\circ}, 8\end{array}$ & 30 & 497,64 \\
\hline
\end{tabular}

Sectionsbefund: Blut hellroth. Herzschlag kräftig. Lungen lufthaltig, hellroth, Leber dito. Thier stirbt ohne Zuckungen.

Tabelle von Serie IV. 
Ueber Oxydation in Warmblüter bei subnormalen Temperaturen. 375

\section{Serie $\mathbf{V}$ (18. Jan.)}

Kaninchen von $1623 \mathrm{gr}$ curarisirt.

Temperatur des Gaszimmers $5^{0}, 0$ C. Barometer 762,0 mm.

Im Laufe der Versuche ergab sich, dass die Gummischläuche der Kaliventile durch den Frost brüchig geworden, weshalb diese Serie keine brauchbaren Resultate ergab.

\section{Serie VI (6. Febr.)}

Kaninchen von $1877 \mathrm{gr}$ curarisirt.

\section{Versuch I.}

Anfang des Versuches $10 \mathrm{Uhr} 43$ Min. Dauer 20 Min.

Zeit . . . $10 \mathrm{Uhr} 43 \mathrm{Min} .48 \quad 53 \quad 11 \mathrm{Uhr} 3 \mathrm{Min}$.

Temperatur des

Thieres . .

39,2

39,0

39,0

$38^{0}, 8$

Temperatur des

Bades . .

38,0

37,0

36,5

$36^{0}, 0$

a) Anfangsvolum auf $0^{\circ}$ und $760 \mathrm{~mm}$ reducirt . . . . $=732,15 \mathrm{ccm}$

b) Endvolum auf $0^{\circ}$ und $760 \mathrm{~mm}$ reducirt , . . . . = 415,85 "

Also Sawerstoffverbrauch in $20 \mathrm{Min}$. . . . . . . . $=316,30$,

Hieraus Sauerstoffverbrauch fïr 1 Kilo und I Stunde

$505,54 \mathrm{ccm}$.

Die mittlere Temperatur des Thieres war

$39^{\circ}, 0 \mathrm{C}$.

Die Kohlensäureproduction konnte nicht bestimmt werden.

\section{Versuch II.}

Anfang des Versuches 11 Uhr 3 Min. Dauer $20 \mathrm{Min}$.

$\begin{array}{llllll}\text { Zeit . . . } & 11 \text { Uhr } 8 \text { Min. } & 13 & 18 & 23\end{array}$

Temperatur des

$\begin{array}{lllll}\text { Thieres . . . } & 38,6 & 38,2 & 38,1 & 37^{0} .9\end{array}$

Temperatur des

Bades . . $\quad 36,0 \quad 35,0 \quad 34,0 \quad 34^{n}, 0$

a) Anfangsvolum auf $0^{\circ}$ und $760 \mathrm{~mm}$ reducirt . . . $=870,65 \mathrm{ccm}$

b) Endvolum auf $0^{\circ}$ und $760 \mathrm{~mm}$ reducirt . . . . . = $=522,88$ "

Also Sauerstoffverbrauch in $20 \mathrm{Min}$. . . . . . . . = $=347,77$ ”

Hieraus Sauerstoffverbrauch für 1 Kilo und 1 Stunde

$555,83 \mathrm{ccm}$.

Die imittlere Temperatur des Thieres war

$38^{\circ}, 2 \mathrm{C}$.

Kohlensäureproduction unbekannt. .

Wegen Benetzung der Gummischläuche mit Kalilauge in Folge fehlerhaften Schlusses der Klemmen wurden fernere Versuche aufgegeben und der Apparat vor Beginn der nächsten Serie ausgespült. 
Tabelle von Serie VI.

\begin{tabular}{|c|c|c|c|c|c|}
\hline $\begin{array}{c}\text { Nummer } \\
\text { des Ver- } \\
\text { suches. }\end{array}$ & $\mid \begin{array}{c}\text { Sauerstoffver- } \\
\text { brauch pro } \\
1 \text { Kilo und } \\
1 \text { Stunde in ccm } \\
\text { auf } 0^{0} \text { und } \\
\text { 0,76M. reducirt. }\end{array}$ & \begin{tabular}{|c} 
Tempera- \\
tur des \\
Thieres, \\
eventuell \\
mittlere.
\end{tabular} & \begin{tabular}{|l} 
Angabe der \\
Grenzen, inner- \\
halb welcher \\
die Temperatur \\
des Thieres \\
schwankte.
\end{tabular} & $\mid \begin{array}{c}\text { Dauer des } \\
\text { einzelnen } \\
\text { Ver- } \\
\text { suches in } \\
\text { Minuten. }\end{array}$ & $\begin{array}{l}\text { Kohlensäure- } \\
\text { ausscheid.ung } \\
\text { pro Kilo und } \\
\text { Stunde in con } \\
\text { auf } 0^{\circ} \text { und } \\
0,76 \mathrm{M} \text {. reducirt }\end{array}$ \\
\hline 1. & 505,54 & $39,0^{\circ} \mathrm{C}$. & Fiel & 20 & unbekannt. \\
\hline 2. & 555,83 & 38,2 n & $\begin{array}{c}\text { Fiel von } 38^{0}, 6 \\
\text { auf } 37^{\circ}, 9 .\end{array}$ & 20 & unbekannt. \\
\hline
\end{tabular}

\section{Serie VII (8. März).}

Kaninchen von $1616 \mathrm{gr}$ curarisirt.

\section{Versuch I.}

Anfang des Versuches $10 \mathrm{Uhr} 11 \mathrm{Min}$. Dauer $20 \mathrm{Min}$.

$\begin{array}{llllll}\text { Zeit . . . . } & 10 \mathrm{Uhr} 11 \mathrm{Min} . & 16 & 21 & 26\end{array}$

Temperatur des

Thieres . . .

Temperatur des

Bades . .

36,8

37,1

37,4

$37^{0}, 5$

a) Anfangsvolum auf $0^{0}$ und $760 \mathrm{~mm}$ reducirt . . . $=716,06 \mathrm{~cm}$

b) Endvolum auf $0^{\circ}$ und $260 \mathrm{~mm}$ reducirt . . . . . = 363,32 "

Also Sauerstoffverbrauch in $20 \mathrm{Min}$. . . . . . . $=352,74$ "

Hieraus Sauerstoffverbrauch für 1 Kilo und 1 Stunde

$$
654,84 \mathrm{ccm} \text {. }
$$

Die mittlere Temperatur des Thieres war

$$
37^{\circ}, 3 \mathrm{C} \text {. }
$$

Die Kohlensäureproduction pro Kilo und Stunde betrug

\section{$728,88 \mathrm{cem}$.}

\section{Versuch II.}

Anfang des Versuches $10 \mathrm{Uhr} 51 \mathrm{Min}$. Dauer $20 \mathrm{Min}$.

$\begin{array}{llllll}\text { Zeit . . . } & 10 \mathrm{Chr} 31 \mathrm{Min} . & 36 & 41 & 46\end{array}$

Temperatur des

$\begin{array}{lllll}\text { Thieres . . . } & 38,0 & 38,1 & 38,1 & 38^{\circ}, 2\end{array}$

Temperatur des

Bades . . . $\quad 38,0 \quad 37,4 \quad 36,8 \quad 36^{\circ}, 8$

a) Anfangsvolum auf $0^{\circ}$ und $760 \mathrm{~mm}$ reducirt . . . $=818,90 \mathrm{ccm}$

b) Endvolum auf $0^{\circ}$ and $760 \mathrm{~mm}$ reducirt . . . . $=441,67$ "

Also Sauerstoffverbrauch in $20 \mathrm{Min}$. . . . . . . $=377,23$ " 
Ueber Oxydation im Warmblüter bei subnormalen Temperaturen. 377

Hieraus Sauerstoffverbrauch für 1 Kilo und 1 Strunde

$700,30 \mathrm{ccm}$

Die mittlere Temperatur des Thieres war

$38^{\circ}, 1 \mathrm{C}$.

Die Kohlensäureproduction pro Kilo und Stunde betrug

$653,33 \mathrm{cem}$.

\section{Versuch III.}

Anfang des Versuches $10 \mathrm{Uhr} 51$ Min. Dauer $20 \mathrm{Min}$.

Zeit . . . $\quad 10 \mathrm{Uhr} 51 \mathrm{Min} . \quad 56 \quad 11 \mathrm{Uhr} 1 \mathrm{Min} . \quad 6$

Temperatur des

$\begin{array}{lllll}\text { Thieres . . . } & 38,2 & 38,2 & 38,2 & 38,{ }^{0} 1\end{array}$

Temperatur des

Bades . . . $\quad 36,5 \quad 36,0 \quad 35,0 \quad 34^{\circ}, 5$

a) Anfangsvolum auf $0^{0}$ und $760 \mathrm{~mm}$ reducirt . . . $=667,70 \mathrm{~cm}$

b) Endvolum auf $0^{\circ}$ and $760 \mathrm{~mm}$ reducirt . . . . . = 287,79

Also Sauerstoffverbrauch in $20 \mathrm{Min}$. . . . . . . $=379,91$ "

Hieraus Sauerstoffverbrauch für 1 Kilo und 1 Stunde

$705,28 \mathrm{ccm}$.

Die mittlere Temperatur des Thieres war

$38^{0}, 2 \mathrm{C}$.

Die Kohlensäureproduction pro Kilo und Stunde betrug

$711,92 \mathrm{ccm}$.

\section{Versuch IV.}

Anfang des Versuches 11 Uhr 11 Min. Dauer 20 Min.

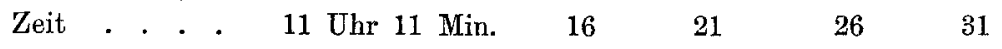

Temperatur des

$\begin{array}{llllll}\text { Thieres . . . } & 38,0 & 37,9 & 37,5 & 37,5 & 37^{\circ}, 4\end{array}$

Temperatur des

Bades . . $\quad 34,5 \quad 34,5 \quad 33,0 \quad 33,0 \quad 32^{0}, 0$

a) Anfangsvolum auf $0^{\circ}$ und $760 \mathrm{~mm}$ reducirt . . . . $=793,46 \mathrm{~cm}$

b) Endvolum auf $0^{\circ}$ and $760 \mathrm{~mm}$ reducirt . . . . = 458,54 " Also Sauerstoffverbrauch in $20 \mathrm{Min}$. . . . . . . . $=334,92$ "

Hieraus Sauerstoffverbrauch für 1 Kilo und 1 Stunde

$621,76 \mathrm{ccm}$.

Die mittlere Temperatur des Thieres war $37^{0}, 6 \mathrm{C}$.

Die Kohlensäureproduction pro Kilo und Stunde betrug $589,43 \mathrm{ccm}$.

Versuch V.

Vor diesem Versuche wurden Thier und Bad abgekühlt. Anfang des Versuches 11 Uhr 55 Min. Dauer 20 Min. 
Zeit . . .

11 Uhr 55 Min.

$12 \mathrm{Uhr}$

5

10

Temperatur des

Thieres . .

Temperatur des

Bades . .

$$
32,5
$$

31,4

29,9

$29^{\circ}, 3$

a) Anfangsvolum auf $0^{\circ}$ und $760 \mathrm{~mm}$ reducirt . . , . $=640,43 \mathrm{ccm}$

b) Endvolum auf $0^{\circ}$ und $760 \mathrm{~mm}$ reducirt . . . . . = 386,65 " Also Sauerstoffverbrauch in 20 Min. . . . . . . . $=253,78$ " Hieraus Sauerstoffverbrauch für 1 Kilo und 1 Stunde

$471,13 \mathrm{ccm}$.

Die mittlere Temperatur des Thieres war $30^{\circ}, 8 \mathrm{C}$.

Die Kohlensäureproduction pro Kilo und Stunde betrug $365,79 \mathrm{ccm}$.

\section{Versuch VI.}

Anfang des Versuches 12 Uhr 15 Min. Dauer 20 Min.

$\begin{array}{lllll}\text { Zeit . . . } & 12 \text { Uhr } 15 \text { Min. } & 20 & 25 & 30\end{array}$

Temperatur des

Thieres . .

Temperatur des

Bades . .

$$
28,1
$$

27,2

26,2

$25^{0}, 3$

a) Anfangsvolum auf $0^{\circ}$ und $760 \mathrm{~mm}$ reducirt . . . $=805,62 \mathrm{ccm}$

b) Endrolum auf $0^{\circ}$ und $760 \mathrm{~mm}$ reducirt . . . . . =642,19 " Also Sauerstoffverbrauch in 20 Min. . . . . . . . $=163,43 "$ Hieraas Sauerstoffverbrauch für 1 Kilo und 1 Stunde

$$
303,40 \mathrm{~mm} \text {. }
$$

Die mittlere Temperatur des Thieres war

$$
26^{0}, 7 \mathrm{C} \text {. }
$$

Die Kohlensäureproduction pro Kilo und Stunde betrug

\begin{tabular}{|c|c|c|c|c|c|}
\hline \multicolumn{6}{|c|}{$\begin{array}{l}\text { Zeit } \cdot \cdot \cdot 12 \text { Uhr } 35 \text { Min. } \\
\text { Temperatur des }\end{array}$} \\
\hline Thicres. . & 24,3 & 23,9 & 23,3 & 22,5 & $22^{0}, 0$ \\
\hline Temperatur de & & & & & \\
\hline Bades & 13,0 & 13,0 & 13,0 & 13,0 & $14^{0}, 0$ \\
\hline
\end{tabular}
$223,27 \mathrm{cem}$.

\section{Versuch VII.}

Anfang des Versuches 12 Uhr 35 Min. Dauex $20 \mathrm{Min}$.

a) Angfangsvolum auf $0^{\circ}$ und $760 \mathrm{~mm}$ reducirt . . . $=644,07 \mathrm{ccm}$

b) Endvolum auf $0^{\circ}$ und $760 \mathrm{~mm}$ reducirt . . . . . =601,27" Also Sauerstoffverbrauch in $20 \mathrm{Min}$. . . . . . . . $=42,80$ " Hieraus Sauerstoffverbrauch für 1 Kilo und 1 Stunde

$79,46 \mathrm{ccm}$. 
Ueber Oxydation im Warmblüter bei subnormalen Temperaturen. 379

Die mittlere Temperatur des Thieres war

$$
23^{\circ}, 2 \mathrm{C} \text {. }
$$

Die Kohlensäureproduction pro Kilo und Stunde betrug

$$
202,77 \mathrm{ccm} \text {. }
$$

Sectionsbefund : Arterialität des Blutes in allen Organen; Herz lebhaft pulsirend. - Während der drei letzten Versuche zuckte das Thier einige Male.

\begin{tabular}{|c|c|c|c|c|c|}
\hline $\begin{array}{c}\text { Nummer } \\
\text { des Ver- } \\
\text { suches. }\end{array}$ & $\mid \begin{array}{c}\text { Sauerstoffver- } \\
\text { brauch pro } \\
1 \text { Kilo und } \\
1 \text { Stunde in cem } \\
\text { auf } 0^{0} \text { und } \\
0,76 \mathrm{M} \text {. reducirt. }\end{array}$ & $\mid \begin{array}{c}\text { Tempera- } \\
\text { tur des } \\
\text { Thieres, } \\
\text { eventuell } \\
\text { mittlere. }\end{array}$ & \begin{tabular}{|c|} 
Angabe der \\
Grenzen, inner- \\
halb welcher \\
die Temperatur \\
des Thieres \\
schwankte.
\end{tabular} & $\begin{array}{l}\text { Dauer des } \\
\text { einzelnen } \\
\text { Ver- } \\
\text { suches in } \\
\text { Minuten. }\end{array}$ & $\begin{array}{l}\text { Kohlensäure- } \\
\text { ausscheidung } \\
\text { pro Kilo und } \\
\text { Stunde in ccm } \\
\text { auf } 0^{\circ} \text { und } \\
0,76 \mathrm{M} \text {. reducirt. }\end{array}$ \\
\hline 1. & 654,84 & $37,3^{\circ} \mathrm{C}$ & Stieg von $36^{n}, 8$ & 20 & 728,88 \\
\hline 2. & 703, & $38,1 n$ & $\begin{array}{l}\text { Stieg von } 38^{\circ}, 0 \\
\text { anf } 38^{\circ}, 2 .\end{array}$ & 20 & 653,33 \\
\hline 3. & 705,28 & 38,2 & $\begin{array}{c}\text { Fiel von } 38^{\circ}, 2 \\
\text { auf } 38^{0}, 1 .\end{array}$ & 20 & 711,92 \\
\hline 4. & 621,76 & 37,6 " & $\mid \begin{array}{c}\text { Fiel von } 38^{\circ}, 0 \\
\text { auf } 37^{\circ}, 4\end{array}$ & 20 & 589,43 \\
\hline 5. & 471,13 & 30,8, & $\mid \begin{array}{c}\text { Fiel von } 32^{\circ}, 5 \\
\text { auf } 29^{\circ}, 3 .\end{array}$ & 20 & 365,79 \\
\hline 6. & 303,40 & 26,7 & $\begin{array}{c}\text { Fiel von } 28^{\circ}, 1 \\
\text { auf } 25^{\circ}, 3 \text {. }\end{array}$ & 20 & 223,27 \\
\hline 7. & 79,46 & $23,2 \%$ & $\mid \begin{array}{c}\text { Fiel von } 24^{0}, 3 \\
\text { auf } 22^{\circ}, 0 .\end{array}$ & 20 & 202,77 \\
\hline
\end{tabular}

Tabelle von Serie VII.

Serie VIII (14. März).

Kaninchen von $2488 \mathrm{gr}$ curarisirt.

\section{Versuch I.}

Anfang des Versuches $10 \mathrm{Uhr} 58 \mathrm{Min}$. Dauer $20 \mathrm{Min}$.

Zeit . . . $10 \mathrm{Uhr} 58 \mathrm{Min} .11 \mathrm{Uhr} 3 \mathrm{Min} .88$

Temperatur des

Thieres . .

Temperatur des

Bades . . .

$$
37,9
$$

38,2

38,5

$38^{0}, 5$

a) Anfangsvolum auf $0^{0}$ und $760 \mathrm{~mm}$ reducirt . . . . $=635,48 \mathrm{ccm}$

b) Endvolum auf $0^{\circ}$ und $760 \mathrm{~mm}$ reducirt . . . . . = $=212,08$, Also Sauerstoffverbrauch in $20 \mathrm{Min}$. . . . . . . . $=423,40$ "

Hieraus Sauerstoffverbrauch für 1 Kilo und 1 Stunde

$$
510,53 \mathrm{ccm} \text {. }
$$

Die mittlere Temperatur des Thieres war

$$
38^{\circ}, 3 \mathrm{C} \text {. }
$$


Die Kohlensätreproduction pro Kilo und Stunde betrug $486,77 \mathrm{cem}$.

\section{Versuch II.}

Anfang des Versuches 11 Uhr 18 Min. Dauer 20 Min.

$\begin{array}{cccccc}\text { Zeit . . . . } & 11 \text { Uhr } 18 \text { Min. } & 23 & 28 & 33 & 38 \\ \begin{array}{c}\text { Temperatur des } \\ \text { Thieres . . . }\end{array} & 38,6 & 38,5 & 38,5 & 38,5 & 38^{\circ}, 5 \\ \begin{aligned} \text { Temperatur des } \\ \text { Bades . . . }\end{aligned} & 38,2 & 37,9 & \mathbf{3 7 , 5} & \mathbf{3 7 , 2} & \mathbf{3 7}, \mathbf{0}\end{array}$

a) Anfangsvolum auf $0^{0}$ und $760 \mathrm{~mm}$ reducirt . . . . $=875,35 \mathrm{ccm}$

b) Endvolum auf $0^{\circ}$ und $760 \mathrm{~mm}$ reducirt . . . . . = 422,74 " Also Sauerstoffverbrauch in $20 \mathrm{Min}$. . . . . . . . $=452,61$ " Hieraus Sauerstoffverbrauch für 1 Kilo und 1 Stunde $545,75 \mathrm{ccm}$.

Die mittlere Temperatur des Thieres war $38^{\circ}, 5$ C.

Die Kohlensäureproduction pro Kilo und Stunde betrug $524,51 \mathrm{ccm}$.

\section{Versueh III.}

Vor diesem Versuche wurden Thier und Bad abgekühlt. Anfang des Versuches 11 Uhr 55 Min. Dauer 20 Min.

Zeit . . . 11 Uhr 55 Min. 12 Uhr $\quad 5 \quad 10$

Temperatur des

$\begin{array}{lllll}\text { Thieres . . . } & 35,2 & 34,2 & 33,2 & 32^{\circ}, 1\end{array}$

Temperatur des

$\begin{array}{lllll}\text { Bades . . . . } & 11,5 & 11,5 & 11,8 & \mathbf{1 1}^{0}, 8\end{array}$

a) Anfangsvolum auf $0^{\circ}$ und $760 \mathrm{~mm}$ reducirt . . . $=644,23 \mathrm{ccm}$

b) Endvolum auf $0^{0}$ und $760 \mathrm{ccm}$ reducirt . . . . . $=291,07$ " Also Sauerstoffverbrauch in $20 \mathrm{Min}$. . . . . . $=353,16$ " Hieraus Sauerstoffverbranch für 1 Kilo und 1 Stunde $425,84 \mathrm{ccm}$.

Die mittlere Temperatur des Thieres war $33^{0}, 1$ C.

Die Kohlensäureproduction pro Kilo und Stunde betrug $428,38 \mathrm{ccm}$.

Versuch IV.

Anfang des Versuches 12 Uhr 15 Min. Dauer 20 Min.

Zeit . . . 12 Uhr 15 Min. $20 \quad 25 \quad 30$

Temperatur des

$\begin{array}{lllll}\text { Thieres . . . } & 30,9 & 30,2 & 29,3 & 28^{\circ}, 6\end{array}$

Temperatur des

Bades

$\begin{array}{llll}11,8 & 11,8 & 11,8 & 1^{\circ}, 8\end{array}$


Ueber Oxydation im Warmbläter bei subnormalen Temperaturen. 381

a) Anfangsvolum auf $0^{\circ}$ und $760 \mathrm{~mm}$ reducirt. . . . $=823,02 \mathrm{ccm}$

b) Endvolum auf $0^{\circ}$ und $760 \mathrm{~mm}$ reducirt . . . . . $=597,63$ "

Also Sauerstoffverbrauch in $20 \mathrm{Min}$. . . . . . . $=225,39$ "

Hieraus Sauerstoffverbrauch für 1 Kilo und 1 Stunde

$271,79 \mathrm{ccm}$.

Die mittlere Temperatur des Thieres war

$29^{\circ}, 3 \mathrm{C}$.

Die Kohlensäureproduction pro Kilo und Stunde betrug

$296,37 \mathrm{cem}$.

Versuch $V$.

Anfang des Versuches 12 Uhr 35 Min. Dauer 25 Min.

Zeit . . . 12 Uhr 35 Min. $40 \quad 45 \quad 50 \quad 55 \quad 1$ Uhr

Temperatur des

$\begin{array}{lllllll}\text { Thieres . . . } & 27,4 & 26,5 & 26,0 & 25,2 & 24,4 & 23^{0}, 7\end{array}$

Temperatur des

$\begin{array}{lllllll}\text { Bades . . . } & 11,8 & 11,8 & 11,8 & 11,9 & 11,9 & 11^{0}, 9\end{array}$

a) Anfangsvolum auf $0^{\circ}$ und $760 \mathrm{~mm}$ reducirt . . . $=600,32 \mathrm{ccm}$

b) Endvolum auf $0^{\circ}$ und $760 \mathrm{~mm}$ reducirt . . . . $=364,60$ "

Also Sauerstoffverbrauch in $25 \mathrm{Min}$. . . . . . . $=235,72$ "

Hieraus Sanerstoffverbrauch für 1 Kilo und 1 Stunde

$227,38 \mathrm{ccm}$.

Die mittlere Temperatur des Thieres war

$25^{\circ}, 5 \mathrm{C}$.

Die Kohlensäureproduction pro Kilo und Stunde betrug:

$269,99 \mathrm{ccm}$.

Versuch VI.

Vor diesem Versuche wurde das Thier in ein warmes Bad gebracht.

Die Temperatur des Thieres vor dem Versuche war $22^{\circ}, 0$.

Anfang des Versuches $1 \mathrm{Uhr} 15 \mathrm{Min}$. Dauer $20 \mathrm{Min}$.

$\begin{array}{llllll}\text { Zeit . . . } & 1 \text { Uhr } 15 \text { Min. } 20 & 25 & 30 & 35\end{array}$

Temperatur des

$\begin{array}{llllll}\text { Thieres . . . } & 22,6 & 23,2 & 24,5 & 25,4 & 27^{\circ}, 0\end{array}$

Temperatur des

Bades . . $\quad 40,0 \quad 40,0 \quad 39,0 \quad 38,5 \quad 38^{\circ}, 0$

a) Anfangsvolum auf $0^{\circ}$ und $760 \mathrm{~mm}$ reducirt . . . $=651,89 \mathrm{ccm}$

b) Endvolum auf $0^{\circ}$ und $760 \mathrm{~mm}$ reducirt . . . . . $=468,74$ "

Also Sanerstoffverbrauch in $20 \mathrm{Min}$. . . . . . . . $=183,15$ "

Hieraus Sauerstoffverbrauch für 1 Kilo and 1 Stunde

$220,84 \mathrm{cam}$.

Die mittlere Temperatur des Thieres war

$24^{0}, 5 \mathrm{C}$.

Die Kohlensäureproduction pro Kilo und Stunde betrug $160,32 \mathrm{eem}$. 


\section{Versuch VII.}

Anfang des Versuches 1 Uhr 35 Min. Dauer 30 Min.

Zeit . . . 1 Uhr 35 Min. $40 \quad 45 \quad 50 \quad 55 \quad 2$ Uhr 2 U. 5 M.

Temperatur des

$\begin{array}{llllllll}\text { Thieres . . } & 27,0 & 27,8 & 29,0 & 29,6 & 30,2 & 30,7 & 31^{0}, 2\end{array}$

Temperatur des

Bades . . $\quad \begin{array}{rrrrrrr}38,0 & 38,0 & 38,0 & 37,5 & 37,0 & 37,0 & 37^{\circ}, 0\end{array}$

a) Anfangsvolum auf $0^{\circ}$ und $760 \mathrm{~mm}$ reducirt . . . $=728,17 \mathrm{ccm}$

b) Endvolum auf $0^{\circ}$ und $760 \mathrm{~mm}$ reducirt . . . . . = 491,02"

Also Sauerstoffverbrauch in $30 \mathrm{Min}$. . . . . . . $=237,15$ "

Hieraus Sauerstoffverbrauch für 1 Kilo und 1 Stunde

$190,64 \mathrm{ccm}$.

Die mittlere Temperatur des Thieres war $29^{\circ}, 5 \mathrm{C}$.

Die Kohlenäureprodaction pro Kilo und Stunde betrug

$$
193,15 \mathrm{ccm} \text {. }
$$

Sectionsbefund wio bei der vorhergehenden Serie.

Tabelle von Serie VIII.

\begin{tabular}{|c|c|c|c|c|c|}
\hline $\begin{array}{l}\text { Nummer } \\
\text { des Ver- } \\
\text { suches. }\end{array}$ & $\begin{array}{l}\text { Sauerstoffver- } \\
\text { brauch pro } \\
1 \text { Kilo und } \\
1 \text { Stunde in ccm } \\
\text { auf } 0^{\circ} \text { und } \\
0,76 \mathrm{M} \text {. reducirt. }\end{array}$ & $\begin{array}{l}\text { Tempera- } \\
\text { tur des } \\
\text { Thieres, } \\
\text { eventuell } \\
\text { mittlere. }\end{array}$ & $\begin{array}{c}\text { A ngabe der } \\
\text { Grenzen, inner- } \\
\text { halb welcher } \\
\text { die Temperatur } \\
\text { des Thieres } \\
\text { schwankte. }\end{array}$ & \begin{tabular}{|} 
Dauer des \\
einzelnen \\
Ver- \\
Suches in \\
Minuten.
\end{tabular} & $\begin{array}{l}\text { Kohlensäure- } \\
\text { ausscheidung } \\
\text { pro Kilo und } \\
\text { Stunde in ccm } \\
\text { auf } 0^{\circ} \text { und } \\
0,76 \mathrm{M} \text {. reducirt. }\end{array}$ \\
\hline 1. & 510,53 & $38,3^{\circ} \mathrm{C}$ & $\begin{array}{l}\text { Stieg von } 37^{\circ}, 9 \\
\quad \text { auf } 38^{\circ}, 6 \text {. }\end{array}$ & 20 & 486,77 \\
\hline 2. & 545,75 & $38,5 n$ & $\begin{array}{l}\text { Fiel von } 38^{\circ}, 6 \\
\text { auf } 38^{0}, 5 \text {. }\end{array}$ & 20 & 524,51 \\
\hline 3. & 425,84 & $33,1 n$ & $\begin{array}{l}\text { Fiel von. } 35^{0}, 2 \\
\text { auf } 30^{\circ}, 9 .\end{array}$ & 20 & 428,38 \\
\hline 4. & 271,79 & $29,3 n$ & $\begin{array}{c}\text { Fiel von } 30^{\circ}, 9 \\
\text { auf } 27^{\circ}, 4\end{array}$ & 20 & 296,37 \\
\hline 5. & 227,38 & & $\begin{array}{l}\text { Fiel von } 27^{\circ}, 4 \\
\text { auf } 23^{\circ}, 7 .\end{array}$ & 25 & 269,99 \\
\hline 6. & 220,84 & $24,5 \#$ & $\begin{array}{l}\text { Stieg von } 22^{0}, 6 \\
\text { auf } 27^{\circ}, 0 .\end{array}$ & 20 & 160,32 \\
\hline 7. & 190,64 & $29,5 n$ & $\begin{array}{l}\text { Stieg von } 27^{\circ}, 0 \\
\text { auf } 31^{\circ}, 2 .\end{array}$ & 30 & 198,15 \\
\hline
\end{tabular}

Kaninchen von $1750 \mathrm{gr}$ curarisirt.

Temperatur des Gaszimmers 70,6. Barometer 754,2.

Während des ersten Versuches wurde durch zu starkes Zusammendrücken des Ballons bei der künstlichen Respiration die Kalilauge aus den Ventilen in die Gummischläuche gedrückt, und deshalb die ferneren Versuche aufgegeben. 
Ueber Oxydation im Warmblïter bei subnormalen Temperaturen. 383

Serie X (21. März).

Kaninchen von $1443 \mathrm{gr}$ curarisirt.

Versueh I.

Anfang des Versuches $9 \mathrm{Uhr} 45$ Min. Dauer 20 Min.

$\begin{array}{ccccc}\text { Zeit . * . } & 9 \text { Uhr } 45 \text { Min. } & 50 & 55 & 10 \mathrm{Uhr} \\ \text { Temperatur des } & & & & \\ \text { Thieres . . } & 37,8 & 38,0 & 38,1 & 38^{\circ}, 2 \\ \text { Temperatur des } & & & & \\ \text { Bades . . . } & \mathbf{3 9 , 5} & \mathbf{3 9 , 5} & \mathbf{3 9 , 0} & \mathbf{3 9}, 0\end{array}$

a) Anfangsvolum auf $0^{\circ}$ und $760 \mathrm{~mm}$ reducirt . . . $=660,53 \mathrm{ccm}$

b) Endvolum auf $0^{\circ}$ und $760 \mathrm{~mm}$ reducirt . . . . . $=373,23$ " Also Sanerstoffverbrauch in $20 \mathrm{Min}$. . . . . . $=287,30$ " Hieraus Sauerstoffverbrauch für 1 Kilo und 1 Stunde

$597,30 \mathrm{ccm}$.

Die mittlere Temperatur des Thieres war

$$
38^{\circ}, 0 \mathrm{C} \text {. }
$$

Die Kohlensäureproduction pro Kilo und Stunde betrug $540,48 \mathrm{cem}$.

\section{Versuch II.}

Anfang des Versuches 10 Uhr 5 Min. Dauer 20 Min.

Zeit . . . $\quad 10$ Uhr 5 Min. $10 \quad 15 \quad 20 \quad 25$

Temperatur des

$\begin{array}{llllll}\text { Thieres . . . } & 38,3 & 38,2 & 38,2 & 38,2 & 38,{ }^{0} 1\end{array}$

Temperatur des

Bades . . . $\quad 38,5 \quad 38,0 \quad 38,0 \quad 38,0 \quad 37^{0}, \mathbf{5}$

a) Anfangsvolum auf $0^{\circ}$ und $760 \mathrm{~mm}$ reducirt . . . . $=781,38 \mathrm{~cm}$

b) Endvolum auf $0^{\circ}$ und $760 \mathrm{~mm}$ reducirt . . . . . = $=467,64 \%$

Also Sauerstoffverbrauch in 20 Min. . . . . . . . $=313,74 \mathrm{ccm}$

Hieraus Sauerstoffverbrauch für 1 Kilo und 1 Stunde

$652,27 \mathrm{ccm}$.

Die mittlere Temperatur des Thieres war

$38^{\circ}, 2 \mathrm{C}$.

Die Kohlensäureproduction pro Kilo und Stunde betrug $515,28 \mathrm{cem}$.

Versuch III.

Vor diesem Versuche wurden Thier und Bad abgekühlt. , Anfang des Versuches $10 \mathrm{Uhr} 40 \mathrm{Min}$. Dauer $20 \mathrm{Min}$.

Zeit . . . 10 Uhr 40 Min. $45 \quad 50 \quad 55$

Temperatur des

$\begin{array}{lllll}\text { Thieres . . . } & 35,3 & 33,6 & 32,2 & 30^{n}, 5\end{array}$

Temperatur des

$\begin{array}{rrrrr}\text { Bades . . . } & 12,0 & 12,0 & 12,0 & 12^{\circ}, 0\end{array}$

E. Pflüger, Archiv f. Physiologie. Bd. XxI. 
a) Anfangsvolum auf $0^{\circ}$ und $760 \mathrm{~mm}$ reducirt . . . . $=669,32 \mathrm{~cm}$

b) Endvolum auf $0^{\circ}$ und $760 \mathrm{~mm}$ reducirt . . . . . = $=419,76$ " Also Sauerstoffverbrauch in $20 \mathrm{Min}$. . . . . . . . $=249,56$ " Hieraus Sauerstoffverbrauch für 1 Kilo und 1 Stunde $518,84 \mathrm{ccm}$.

Die mittlere Temperatur des Thieres war $32^{\circ}, 9 \mathrm{C}$.

Die Kohlensäureproduction pro Kilo und Stunde $414,26 \mathrm{ccm}$.

\section{Versuch IV.}

Anfang des Versuches 11 Uhr. Dauer 20 Min.

$\begin{array}{ccccc}\text { Zeit . . . } & 11 \text { Uhr } & 5 & 10 & 15 \\ \text { Temperatur des } & & & & \\ \text { Thieres . . } & 29,3 & 28,0 & 26,7 & 25^{\circ}, 7 \\ \begin{array}{r}\text { Temperatur des } \\ \text { Bades . . . }\end{array} & 12,0 & 12,0 & 12,0 & 12^{\circ}, 0\end{array}$

a) Anfangsvolum auf $0^{\circ}$ und $760 \mathrm{~mm}$ reducirt . . . $=782,39 \mathrm{ccm}$

b) Endvolum auf $0^{\circ}$ und $760 \mathrm{~mm}$ reducirt . . . . . = $=671,26$ " Also Sauerstoffverbrauch in $20 \mathrm{Min}$. . . . . . . . $=111,13$ " Hieraus Sauerstoffverbrauch für 1 Kilo und 1 Stunde $231,03 \mathrm{ccm}$.

Die mittlere Temperatur des Thieres war $27^{\circ}, 4 \mathrm{C}$.

Die Kohlensäureproduction pro Kilo und Stunde betrug $245,24 \mathrm{eem}$.

\section{Versuch $V$.}

Anfang des Versuches 11 Uhr 20 Min. Daner 20 Min.

Zeit . . . 11 Uhr 20 Min. $25 \quad 30 \quad 35 \quad 40$

Temperatur des

Thieres . .

Temperatur des

$$
24,7 \quad 24,0 \quad 23,2 \quad 22,0 \quad 21^{0}, 1
$$

Bades . .

$11,5 \quad 11,5 \quad 11,5 \quad 11,5 \quad 11^{0}, 5$

a) Anfangsvolum auf $0^{0}$ und $760 \mathrm{~mm}$ reducirt . . . $=621,54 \mathrm{ccm}$

b) Endvolum auf $0^{\circ}$ und $760 \mathrm{~mm}$ reducirt . . . . . $=455,18$ " Also Sauerstoffverbrauch in 20 Min. . . . . . . . $=166,36$ " Hieraus Sanerstoffverbrauuch für 1 Kilo und 1 Stunde $345,89 \mathrm{ccm}$

Die mittlere Temperatur des Thieres war $23^{0}, 2 \mathrm{C}$.

Die Kohlensätreproduction pro Kilo und Stunde betrug $185,26 \mathrm{ccm}$. 
Ueber Oxydation im Warmblüter bei subnormalen Temperaturen. 385

\section{Versuch VI.}

Vor diesem Versuche wurde das Thier in ein warmes Bad gebracht; während des Wechselns fiel die Temperatur des Thieres auf $20^{\circ}, 4$.

Anfang des Versuches 11 Uhr 50 Min. Dauer 20 Min.

$\begin{array}{ccccc}\text { Zeit . . . } & 11 \text { Uhr } 50 \text { Min. } & 55 & 12 \text { Uhr } & 5 \\ \text { Temperatur des } & & & & \\ \text { Thieres . . . } & 20,6 & 21,7 & 23,5 & 25^{0}, 3 \\ \text { Temperatur des } & & & & \\ \text { Bades . . - } & 40,0 & 39,5 & 39,0 & 39^{\circ}, 0\end{array}$

a) Anfangsvolum auf $0^{0}$ und $760 \mathrm{~mm}$ reducirt . . . $=755,31 \mathrm{ccm}$

b) Endvolum auf $0^{\circ}$ und $760 \mathrm{~mm}$ reducirt . . . . . $=686,14$ "

Also Sauerstoffverbrauch in $20 \mathrm{Min}$. . . . . . . . $=69,17$ "

Hieraus Sauerstoffverbrauch für 1 Kilo und 1 Stunde

$143,80 \mathrm{~cm}$.

Die mittlere Temperatur des Thieres war

$22,05 \mathrm{C}$.

Die Kohlensäureproduction pro Kilo und Stunde betrug $149,69 \mathrm{ccm}$.

Versuch VII.

Anfang des Versuches 12 Uhr 10 Min. Dauer 30 Min.

Zeit . . . 12 Uhr 10 Min. $15 \quad 20 \quad 25$

Temperatur des

$\begin{array}{lllll}\text { Thieres . . . } & 27,3 & 28,5 & 29,8 & 30^{\circ}, 0\end{array}$

Temperatur des

Bades . . . $\quad 39,0 \quad 38,5 \quad 38,5 \quad 38^{\circ}, 0$

a) Anfangsvolum auf $0^{0}$ und $760 \mathrm{~mm}$ reducirt . . . $=633,45 \mathrm{ccm}$

b) Endvolum auf $0^{\circ}$ und $760 \mathrm{~mm}$ reducirt . . . . . $=529,12$ n

Also Sauerstoffverbrauch in $20 \mathrm{Min}$. . . . . . . $=104,33$,

Hieraus Sauerstoffverbrauch für 1 Kilo und 1 Stunde

$216,90 \mathrm{cem}$.

Die mittlere Temperatur des Thieres wár

$28^{\circ}, 9$ C.

Die Kohlensäureproduction pro Kilo und Stunde betrug

$184,24 \mathrm{ccm}$.

\section{Versuch VIII.}

Anfang des Versuches 12 Uhr $30 \mathrm{Min}$. Dauer $25 \mathrm{Min}$.

$\begin{array}{lllllll}\text { Zeit . . } 12 \text { Uhr } 30 \text { Min. } 35 & 40 & 45 & 50 & 55\end{array}$

Temperatur des

Thieres. . .

Temperatur des

Bades . .

$31,5 \quad 32,0 \quad 32,6 \quad 33,1 \quad 33,5 \quad 34^{0}, 0$

$38,0 \quad 38,5 \quad 39,0 \quad 39,0 \quad 39,0 \quad 38^{\circ}, 5$ 
a) Anfangsvolum auf $0^{\circ}$ und $760 \mathrm{~mm}$ reducirt . . . $=800,76 \mathrm{~cm}$

b) Endrolum auf $0^{0}$ und 761$) \mathrm{mm}$ reducirt . . . . . = $=661,99$ " Also Sauerstoffverbrauch in $25 \mathrm{Min}$. . . . . . . . $=138,77$ n

Hieraus Sauerstoffverbrauch für 1 Kilo und 1 Stunde $230,80 \mathrm{ccm}$.

Die mittlere Temperatur des Thieres war

$$
32^{0}, 8 \mathrm{C} \text {. }
$$

Die Kohlensäureproduction pro Kilo und Stunde betrug

$$
258,17 \mathrm{ccm} \text {. }
$$

Sectionsbefund: Sehr lebhäft pulsirendes Herz. Blut hellroth.

\begin{tabular}{|c|c|c|c|c|c|}
\hline $\begin{array}{c}\text { Nummer } \\
\text { des Ver- } \\
\text { suches. }\end{array}$ & $\mid \begin{array}{c}\text { Sauerstoffver- } \\
\text { branch pro } \\
1 \text { Kilo und } \\
1 \text { Stunde in com } \\
\text { auf } 0^{\circ} \text { und } \\
0,76 \mathrm{M} \text {. reducirt. }\end{array}$ & $\mid \begin{array}{c}\text { Tempera } \\
\text { tur des } \\
\text { Thieres } \\
\text { eventuell } \\
\text { mittlere. }\end{array}$ & \begin{tabular}{|c||} 
Angabe der \\
Grenzen, inner- \\
halb welcher \\
die Temperatur \\
des Thieres \\
schwankte.
\end{tabular} & \begin{tabular}{|} 
Dauer des \\
einzelnen \\
Ver- \\
suches in \\
Minuten.
\end{tabular} & $\begin{array}{l}\text { Kohlensäure- } \\
\text { ausscheidung } \\
\text { pro Kilo und } \\
\text { Stunde in ccm } \\
\text { auf } 0^{\circ} \text { und } \\
\text { 0,76M. reducirt }\end{array}$ \\
\hline 1. & 597,30 & $38,0^{\circ} \mathrm{C}$ & Stieg von $37^{\circ}, 8$ & 20 & 540,48 \\
\hline 2. & 652,17 & 38,2 " & $\begin{array}{c}\text { Fiel von } 38^{\circ}, 3 \\
\text { aaf } 38^{\circ}, 1 .\end{array}$ & 20 & 515,28 \\
\hline 3. & 518,84 & & $\begin{array}{c}\text { Fiel von } 35^{n}, 3 \\
\text { auf } 30^{\circ}, 5\end{array}$ & 20 & 414,26 \\
\hline 4. & 231,0 & 27,4 & $\begin{array}{l}\text { Fiel von } 29^{\circ}, 3 \\
\text { auf } 25^{\circ}, 7 .\end{array}$ & 20 & 245,24 \\
\hline 5. & 345,89 & 23,2 " & $\mid \begin{array}{c}\text { Fiel von } 24^{0}, 7 \\
\text { auf } 21^{\circ}, 1^{1}\end{array}$ & 20 & 185,26 \\
\hline 6. & 143,80 & 22,5 & $\begin{array}{c}\text { Stieg von } 20^{\circ}, 6 \\
\text { auf } 25^{\circ} 3 .\end{array}$ & 20 & 149,69 \\
\hline 7. & 216,90 & 28,9 & $\begin{array}{c}\text { Stieg von } 27^{\circ}, 3 \\
\text { auf } 30^{\circ}, 0 .\end{array}$ & 20 & 184,24 \\
\hline 8. & 230,80 & 32,8, & $\mid \begin{array}{c}\text { Stieg von } 31^{\circ}, 5 \\
\text { auf } 34^{\circ}, 0 .\end{array}$ & 25 & 258,17 \\
\hline
\end{tabular}

Tabelle von Serie X.

Serie XI (25. März).

Kaninchen von $2159 \mathrm{gr}$ curarisirt.

\section{Versuch I.}

Anfang des Versuches 9 Uhr 45 Min. Dauer 20 Min.

Zeit . . . $9 \mathrm{Uhr} 45 \mathrm{Min} . \quad 50 \quad 55 \quad 10 \mathrm{Uhr}$

Temperatur des

Thieres . .

Temperatur des

Bades . . .

$$
37,5
$$$$
37,8 \quad 38,0
$$

$38^{\circ}, 2$

a) Anfangsvolum auf $0^{\circ}$ and $760 \mathrm{~mm}$ reducirt . . . . $=624,25 \mathrm{~cm}$

b) Endvolum auf $0^{\circ}$ und $760 \mathrm{~mm}$ reducirt . . . . . = 305,92 "

Also Sauerstoffverbrauch in $20 \mathrm{Min}$. . . . . . $=318,33$ " 
Ueber Oxydation im Warmblüter bei subnormalen Temperaturen. 387

Hieraus Sauerstoffverbrauch für 1 Kilo und 1 Stunde

$442,33 \mathrm{ccm}$.

Die mittlere Temperatur des Thieres war

$37^{\circ}, 9 \mathrm{C}$.

Die Kohlensäureproduction pro Kilo und Stunde betrug

$431,71 \mathrm{ccm}$.

\section{Versuch II.}

Anfang des Versuches $10 \mathrm{Uhr} 5 \mathrm{Min}$. Dauer $20 \mathrm{Min}$.

$\begin{array}{lllllll}\text { Zeit . . . } & 10 \text { Uhr } 5 \text { Min. } & 10 & 15 & 20 & 25\end{array}$

Temperatur des

$\begin{array}{llllll}\text { Thieres . . } & 38,2 & 38,4 & 38,2 & 38,2 & 38^{\circ}, 1\end{array}$

Temperatur des

Bades . . . $\quad 38,0 \quad 38,0 \quad 37,5 \quad 37,0 \quad 37^{\circ}, 0$

a) Anfangsvolum auf $0^{\circ}$ und $760 \mathrm{~mm}$ reducirt . . . $=783,40 \mathrm{ccm}$

b) Endvolum auf $0^{\circ}$ and $760 \mathrm{~mm}$ reducirt . . . . . = =404,03"

Also Sauerstoffverbrauch in $20 \mathrm{Min}$. . . . . . . $=379,37$ "

Hieraus Sauerstoffverbrauch für 1 Kilo und 1 Stunde

$527,15 \mathrm{ccm}$.

Die mittlere Temperatur des Thieres war

$38^{\circ}, 2 \mathrm{C}$.

Die Kohlensäureproduction pro Kilo betrug

$519,56 \mathrm{ccm}$.

\section{Versnch III.}

Vor diesem Versuche wurde das abgekühlte Thier in ein kälteres Bad gebracht.

Anfang des Versuches $10 \mathrm{Uhr} 45 \mathrm{Min}$. Dauer $20 \mathrm{Min}$.

Zeit . . . 10 Uhr 45 Min. $50 \quad 55 \quad 11 \mathrm{Uhr}$

Temperatur des

Thieres . . .

$34,6 \quad 33,5 \quad 32,5 \quad 30^{\prime \prime}, 8$

Temperatur des

Bades . . . $\quad 11,0 \quad 11,0 \quad 11,0 \quad 10^{0}, 5$

a) Anfangsvolum auf $0^{\circ}$ und $760 \mathrm{~mm}$ reducirt . . . $=643,60 \mathrm{cem}$

b) Endvolum auf $0^{\circ}$ and $760 \mathrm{~mm}$ reducirt . . . . . $=396,44$ "

Also Sauerstoffverbrauch in $20 \mathrm{Min}$. . . . . . . . $=247,16$ "

Hieraus Sauerstoffverbrauch für 1 Kilo und 1 Stunde

$343,44 \mathrm{ccm}$.

Die mittlere Temperatur des Thieres war

$32^{\circ}, 2 \mathrm{C}$.

Die Kohlensäureproduction pro Kilo and Stunde betrug

$371,82 \mathrm{ecm}$. 


\section{Versuch IV.}

Anfang des Versuches 11 Uhr 5 Min. Dauer 25 Min.

Zeit . . . . 11 Uhr 5 Min. $10 \quad 15 \quad 20 \quad 25 \quad 30$

Temperatur des

Thieres . . .

Temperatur des

$\begin{array}{llllll}29,6 & 28,6 & 27,7 & 26,7 & 25,5 & 21^{0}, 5 \\ 10,0 & 10,0 & 10,0 & 10,0 & 10,0 & 10^{\circ}, 0\end{array}$
Bades . . . $\quad 10,0 \quad 10,0 \quad 10,0 \quad 10,0 \quad 10,0 \quad 10^{\circ}, 0$
a) Anfangsvolum auf $0^{\circ}$ und $760 \mathrm{~mm}$ reducirt . . . $=793,72 \mathrm{ccm}$
b) Endvolum auf $0^{\circ}$ und $760 \mathrm{~mm}$ reducirt . . . . = $=593,80$ " Also Sauerstoffverbrauch in $25 \mathrm{Min}$. . . . . . . $=199,92$ "
Hieraus Sauerstoffverbrauch für 1 Kilo und 1 Stunde
$222,69 \mathrm{ccm}$.

Die mittlere Temperatur des Thieres war

$27^{\circ}, 1 \mathrm{C}$.

Die Kohlensäureproduction pro Kilo und Stunde betrug

$229,46 \mathrm{ccm}$.

\section{Versuch V.}

Yor diesem Versuche wurde das Thier in ein warmes Bad gebracht.

Anfang des Versuches 11 Uhr 45 Min. Dauer 20 Min.

Zeit . . . 11 Uhr 45 Min. $50 \quad 55 \quad 12$ Uhr 5

Temperatur des

Thieres. .

Temperatur des

Bades . . $\quad 45,0 \quad 44,0 \quad 43,5 \quad 43,0 \quad 43^{0}, 0$

a) Anfangsvolum auf $0^{\circ}$ und $760 \mathrm{~mm}$ reducirt . . . $=647,75 \mathrm{ccm}$

b) Endvolum auf $0^{\circ}$ und $760 \mathrm{~mm}$ reducirt . . . . . = 506,33

Also Sauerstoffverbrauch in $20 \mathrm{Min}$. . . . . . . $=141,42$ "

Hieraus Sauerstoffverbrauch für 1 Kilo und 1 Stunde

$196,51 \mathrm{ccm}$.

Die mittlere Temperatur des Thieres war

$$
26^{\circ}, 2 \mathrm{C} \text {. }
$$

Die Kohlensäureproduction pro Kilo und Stunde betrug $106,27 \mathrm{cem}$.

Es wurde noch ein IV. Versuch während 20 Minuten angestellt, aber es ergab sich am Ende desselben, dass das Thier keinen Sanerstoff mehr verbraucht hatte, also todt war. Bei der Section ergab sich, dass beim letzten Wechseln in Folge von Verletzung durch die Tracheacanüle eine Blutung in die Luftwege stattgefunden, woran das Thier erstickt war; dunkles Blut; kein Herzschlag; Lungen lufthaltig. 
Ueber 0xydation im Warmblüter bei subnormalen Temperaturen. 389

Tabelle von-Serie XI.

\begin{tabular}{|c|c|c|c|c|c|}
\hline $\begin{array}{l}\text { Nummer } \\
\text { des Ver- } \\
\text { suches. }\end{array}$ & $\begin{array}{l}\text { Sauerstoffer- } \\
\text { brauch pro } \\
1 \text { Kilo und } \\
1 \text { Stunde in ccm } \\
\text { auf } 0^{\circ} \text { und } \\
0,76 \mathrm{M} \text {. reducirt. }\end{array}$ & $\begin{array}{l}\text { Tempera- } \\
\text { tur des } \\
\text { Thieres } \\
\text { eventuell } \\
\text { mittlere }\end{array}$ & $\begin{array}{c}\text { Angabe der } \\
\text { Grenzen, inner- } \\
\text { halb welcher } \\
\text { die Temperatur } \\
\text { des Thieres } \\
\text { schwankte. }\end{array}$ & $\begin{array}{l}\text { Dauer des } \\
\text { einzelnen } \\
\text { Ver- } \\
\text { suches in } \\
\text { Minuten. }\end{array}$ & $\begin{array}{l}\text { Kohlensäure- } \\
\text { ausscheilutug } \\
\text { pro Kilo und } \\
\text { Stunde in com } \\
\text { auf } 0^{\circ} \text { and } \\
0,76 \mathrm{M} \text {. reducirt. }\end{array}$ \\
\hline 1. & 442,33 & $37,9^{\circ} \mathrm{C}$ & Stieg von $37^{\circ}, 5$ & 20 & 431,71 \\
\hline 2. & 527,15 & 38,2 & Stieg von $38^{\circ}, 2$ & 20 & 519,56 \\
\hline 3. & 343,44 & & $\mid \begin{array}{c}\text { Fiel von } 34^{\circ}, 6 \\
\text { auf } 29^{\circ}, 6 .\end{array}$ & 20 & 371,82 \\
\hline 4. & 222,69 & 27,1 & $\begin{array}{c}\text { Fiel von } 29^{\circ}, 6 \\
\text { auf } 24^{\circ}, 5 \text {. }\end{array}$ & 25 & 229,46 \\
\hline & 196,51 & 26,2 " & $\mid \begin{array}{c}\text { Stieg von } 23^{\circ}, 7 \\
\text { auf } 29^{\circ}, 0 .\end{array}$ & 20 & 106,27 \\
\hline 6. & Thier todt. & & & & \\
\hline
\end{tabular}

Serie XII (26. März).

Kaninchen von $2214 \mathrm{gr}$ curarisirt.

\section{Versuch I.}

Anfang des Versuches 11 Uhr 35 Min. Dauer 20 Min.

Zeit . . $\quad 11$ Uhr 35 Min. $40 \quad 45 \quad 50 \quad 55$

Temperatur des

$\begin{array}{llllll}\text { Thieres . . . } & 37,2 & 37,3 & 37,4 & 37,5 & 37^{0}, 4\end{array}$

Temperatur des

Bades . . . $\quad 38,0 \quad 38,5 \quad 38,0 \quad 37,0 \quad 37^{\circ}, 5$

a) Anfangsvolum auf $0^{\circ}$ und $760 \mathrm{~mm}$ reducirt . . . $=667,68 \mathrm{ccm}$

b) Endvolum auf $0^{\circ}$ und $760 \mathrm{~mm}$ reducirt . . . . . = $=242,06$ "

Also Sauerstoffverbrauch in $20 \mathrm{Min}$. . . . . . . $=425,62 "$

Hieraus Sauerstoffverbrauch für 1 Kilo und 1 Stunde

$576,72 \mathrm{ccm}$.

Die mittlere Temperatur des Thieres war

$37^{\circ}, 4 \mathrm{C}$.

Die Kohlensäureproduction pro Kilo und Stunde betrug $524,17 \mathrm{ecm}$.

Versuch II.

Anfang des Versuches 11 Uhr 55 Min. Dauer 20 Min.

Zeit . . . 11 Uhr 55 Min. 12 Uhr $\quad 5 \quad 10 \quad 15$

$\begin{array}{lllll}\text { Temperatur des Thieres } & 37,3 & 37,4 & 37,5 & 37^{\circ}, 4\end{array}$

$\begin{array}{lllll}\text { Temperatur des Bades } & 37,0 & 38,0 & 37,0 & 37^{\circ}, 5\end{array}$ 
a) Anfangsvolum auf $0^{\circ}$ und $760 \mathrm{~mm}$ reducirt . . . . $=805,04 \mathrm{ccm}$

b) Endvolum auf $0^{\circ}$ und $760 \mathrm{~mm}$ reducirt . . . . . = 438,87" Also Sauerstoffverbrauch in $20 \mathrm{Min}$. . . . . . . $=366,17$ " Hieraus Sauerstoffverbrauch für 1 Kilo und 1 Stunde $496,17 \mathrm{ccm}$.

Die mittlere Temperatur des Thieres war $37^{\circ}, 3 \mathrm{C}$.

Die Kohlensäureproduction pro Kilo und Stunde betrug $430,13 \mathrm{cem}$.

\section{Versuch III.}

Vor diesem Versuche wurden Thier und Bad abgekühlt. Anfang des Versuches 1 Uhr 5 Min. Dauer 20 Min.

$\begin{array}{lllllll}\text { Zeit . . . } & 1 & \text { Uhr } 5 \text { Min. } & 10 & 15 & 20 & 25\end{array}$

Temperatur des

Thieres . . 25,7 $25,4 \quad 24,9 \quad 24,5$ $24^{0}, 2$

Temperatur des

$\begin{array}{llllll}\text { Bades . . . } & 19,5 & 19,0 & 19,0 & 19,0 & 19^{\circ}, 0\end{array}$

a) Anfangsvolum auf $0^{\circ}$ und $760 \mathrm{~mm}$ reducirt . . . $=656,04 \mathrm{ccm}$

b) Endvolum auf $0^{\circ}$ und $760 \mathrm{~mm}$ reducirt . . . . . $=502,27$ " Also Sauerstoffverbrauch in $20 \mathrm{Min}$. . . . . . . . $=153,77$ " Hieraus Sauerstoffverbrauch für 1 Kilo und 1 Stunde $208,36 \mathrm{ccm}$.

Die mittlere Temperatur des Thieres war $24^{\circ}, 9 \mathrm{C}$.

Die Kohlensäureproduction pro Kilo und Stunde betrug $220,42 \mathrm{ccm}$.

\section{Versuch IV.}

Anfang des Versuches 1 Uhr 25 Min. Dauer $20 \mathrm{Min}$.

$\begin{array}{lllllll}\text { Zeit . . . } & 1 \text { Uhr } 25 \text { Min. } & 30 & 35 & 40 & 45\end{array}$

Temperatur des

Thieres . .

$23,7 \quad 23,2 \quad 23,0 \quad 22^{0}, 7$

Temperatur des

Bades . . .

$19,0 \quad 19,0 \quad 19,0 \quad 19^{9}, 0$

a) Anfangsvolum auf $0^{\circ}$ und $760 \mathrm{~mm}$ reducirt . . . $=847,21 \mathrm{ccm}$

b) Endvolum auf $0^{\circ}$ und $760 \mathrm{~mm}$ reducirt . . . . . = $=731,73$ " Also Sauerstoffverbrauch in $20 \mathrm{Min}$. . . . . . . . $=115,48$ " Hieraus Sauerstoffverbrauch für 1 Kilo und 1 Stunde

$156,48 \mathrm{ccm}$.

Die mittlere Temperatur des Thieres war $23^{0}, 4$ C.

Die Kohlensäureproduction pro Kilo und Stunde betrug $175,27 \mathrm{ccm}$. 
Ueber Oxydation im Warmblüter bei subnormalen Temperaturen. 391

\section{Versuch V.}

Vor diesem Versuche wurde die Temperatur des Thieres durch Uebergiessen von warmem Wasser erhöht und dasselbe in ein warmes Bad gebracht.

Anfang des Versuches $2 \mathrm{Uhr} 57$ Min. Dauer $20 \mathrm{Min}$.

$\begin{array}{lllllll}\text { Zeit . . . } 2 \text { Uhr } 57 \text { Min. } 3 \text { U. } 2 \text { M. } 7 & 12 & 17\end{array}$

Temperatur des

$\begin{array}{llllll}\text { Thieres . . . } & 36,0 & 36,1 & 36,2 & 36,2 & 36^{0}, 2\end{array}$

Temperatur des

Bades . . . $\quad 39,0 \quad 38,5 \quad 38,5 \quad 38,0 \quad 38^{\circ}, 0$

a) Anfangsvolum anf $0^{\circ}$ und $760 \mathrm{~mm}$ reducirt . . . = $=702,04 \mathrm{ccm}$

b) Endvolum auf $0^{\circ}$ und $760 \mathrm{~mm}$ reducirt . . . . . $=408,99$ "

Also Sauerstoffverbrauch in $20 \mathrm{Min}$. . . . . . . $=298,05$ "

Hieraus Sauerstoffverbrauch für 1 Kilo und 1 Stunde

$403,86 \mathrm{ccm}$.

Die mittlere Temperatur des Thieres war

$36^{\circ}, 2 \mathrm{C}$.

Die Kohlensäureproduction pro Kilo und Stunde betrug

391,65 cem.

\section{Versuch VI.}

Anfang des Versuches 3 Uhr 17 Min. Dauer 20 Min.

$\begin{array}{llllllll}\text { Zeit . . . } & 3 & \text { Uhr } 17 & \text { Min. } & 22 & 27 & 32 & 37\end{array}$

Temperatur des

Thieres. . .

$36,0 \quad 36,0 \quad 35,9 \quad 35^{0}, 8$

Temperatur des

Bades . . $\quad 37,0 \quad 37,0 \quad 37,0 \quad 36^{0}, 0$

a) Anfangsvolum auf $0^{\circ}$ und $760 \mathrm{~mm}$ reducirt . . . $=862,74 \mathrm{~cm}$

b) Endvolum auf $0^{\circ}$ und $760 \mathrm{~mm}$ reducirt . . . . . $=569,56$ "

Also Sauerstoffverbrauch in $20 \mathrm{Min}$. . . . . . . . $=293,18$ "

Hieraus Sauerstoffverbrauch für 1 Kilo und 1 Stunde

$397,26 \mathrm{~cm}$.

Die mittlere Temperatur des Thieres war

$36^{\circ}, 0$ C.

Die Kohlensäureproduction pro Kilo und Stunde betrug $392,22 \mathrm{cem}$.

Sectionsbefund: Starker Herzschlag. Arterialität des Blutes in allen Organen. Lungen lufthaltig. 
Tabelle von Serie XII.

\begin{tabular}{|c|c|c|c|c|c|}
\hline $\begin{array}{l}\text { Nummer } \\
\text { des Ver- } \\
\text { suches. }\end{array}$ & $\begin{array}{l}\text { Sauerstoffver- } \\
\text { brauch pro } \\
1 \text { Kilo und } \\
1 \text { Stunde in cem } \\
\text { auf } 0^{\circ} \text { und } \\
0,76 \mathrm{M} \text {. reducirt. }\end{array}$ & $\begin{array}{l}\text { Tempera- } \\
\text { tur des } \\
\text { Thieres } \\
\text { eventuell } \\
\text { mittlere. }\end{array}$ & $\begin{array}{l}\text { Angabe der } \\
\text { Grenzen, inner-| } \\
\text { halb welcher } \\
\text { die Temperatur } \\
\text { des Thieres } \\
\text { schwankte. }\end{array}$ & \begin{tabular}{|} 
Dauer des \\
einzelnen \\
Ver- \\
suches in \\
Minuten.
\end{tabular} & $\begin{array}{l}\text { Kohlensäure- } \\
\text { abscheidung } \\
\text { pro Kilo und } \\
\text { Stunde in cem } \\
\text { auf } 0^{\circ} \text { und } \\
0,76 \mathrm{M} \text {. reducirt. }\end{array}$ \\
\hline 1. & 576,72 & $37,4^{\circ} \mathrm{C}$ & $\mid \begin{array}{r}\text { Stieg von } 37^{0}, 2 \\
\text { auf } 37^{0}, 5 \text { und } \\
\text { fiel }\end{array}$ & 20 & 524,17 \\
\hline 2. & 496,17 & $37,3 n$ & $\begin{array}{c}\text { Fiel von } 37^{\circ}, 4 \\
\text { auf } 37^{\circ}, 2 .\end{array}$ & 20 & 430,13 \\
\hline 3. & 208,36 & & $\begin{array}{c}\text { Fiel von } 25^{\circ}, 7 \\
\text { auf } 24^{\circ}, 2 .\end{array}$ & 20 & 220,12 \\
\hline 4. & 156,48 & 23,4 & $\begin{array}{l}\text { Fiel von } 24^{\circ}, 2 \\
\text { auf } 22^{0}, 7\end{array}$ & 20 & 175,27 \\
\hline 5. & 403,86 & & $\begin{array}{l}\text { Stieg von } 36^{\circ}, 0 \\
\text { auf } 36^{0}, 2 .\end{array}$ & 20 & 391,65 \\
\hline 6. & 397,26 & 36,0, & $\mid \begin{array}{c}\text { Fiel von } 36^{\circ}, 2 \\
\text { auf } 35^{\circ}, 8 .\end{array}$ & 20 & 392,22 \\
\hline
\end{tabular}

Bei der Aufstellung der aus diesen Versuchsreihen sich ergebenden Generaltabellen zur Feststellung der Mittelwerthe, erschien es zweckmässig, nach den verschiedenen Temperaturen fünf Stufen anzunehmen, deren erste die Temperaturen von 39,0-37,9 (Mittel 38,33 ), die zweite die von 37,7-37,2 (Mittel 37,41), die dritte die von 33,1-28,8 (Mittel 31,37), die vierte die von 27,8-24,5 (Mittel 26,17), die fünfte die von 23,4-22,5 (Mittel 23,07) umfasste. Ausserdem wurden für die nach geschehener Abkühlung wieder aufsteigenden Temperaturen noch zwei Stufen gebildet; in der sechsten sind die Temperaturen von 28,9-32,8 (Mittel 30,44), in der siebenten die von 36,0-36,7 (Mittel 36,37) enthalten. 
Ueber Oxydation im Warmblüter bei subnormalen Temperaturen. 393

Generaltabelle I.

\begin{tabular}{|c|c|c|c|c|}
\hline $\begin{array}{l}\text { Versuchs- } \\
\text { nummer. }\end{array}$ & $\begin{array}{l}\text { Sauerstoff } \\
\text { in cem. }\end{array}$ & $\begin{array}{l}\text { Temperatur } \\
\text { in Recto (C.). }\end{array}$ & $\begin{array}{l}\text { Dauer des Ver- } \\
\text { suches in Min. }\end{array}$ & $\begin{array}{l}\text { Kohlensäure } \\
\text { in ccm. }\end{array}$ \\
\hline 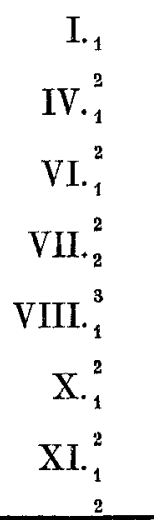 & $\begin{array}{l}616,69 \\
704,76 \\
506,12 \\
534,59 \\
505,54 \\
555,83 \\
700,30 \\
705,28 \\
510,53 \\
545,75 \\
597,30 \\
652,17 \\
442,33 \\
527,15 \\
\end{array}$ & $\begin{array}{r}38,4 \\
38,9 \\
38,3 \\
38,3 \\
39,0 \\
38,2 \\
38,1 \\
38,2 \\
38,3 \\
38,5 \\
38,0 \\
38,2 \\
37,9 \\
38,2 \\
\end{array}$ & $\begin{array}{l}20 \\
25 \\
20 \\
20 \\
20 \\
20 \\
20 \\
20 \\
20 \\
20 \\
20 \\
20 \\
20 \\
20 \\
\end{array}$ & $\begin{array}{c}827,03 \\
644,93 \\
487,87 \\
487,72 \\
- \\
- \\
653,33 \\
711,92 \\
486,77 \\
524,51 \\
540,48 \\
515,28 \\
431,71 \\
519,56 \\
\end{array}$ \\
\hline Summe: & 16561,06 & 1092,45 & $285(245)$ & $139 \overline{94,685}$ \\
\hline Mittel: & 581,09 & $\begin{array}{c}38,33 \\
\text { für Sauersto } \\
38,29 \\
\text { iur Kohlensäu } \\
\text { torischer Qu }\end{array}$ & nt 0,98 & 570,80 \\
\hline
\end{tabular}

Generaltabelle II.

\begin{tabular}{|c|c|c|c|c|}
\hline $\begin{array}{l}\text { Versuchs- } \\
\text { nummer. }\end{array}$ & $\begin{array}{l}\text { Sauerstoff } \\
\text { in ccm. }\end{array}$ & $\begin{array}{l}\text { Temperatur } \\
\text { in Recto (C.). }\end{array}$ & $\begin{array}{c}\text { Dauer des Ver- } \\
\text { suches in Min. }\end{array}$ & $\begin{array}{l}\text { Kohlensäure } \\
\text { in ccm. }\end{array}$ \\
\hline 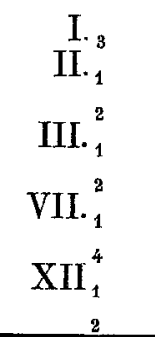 & $\begin{array}{l}557,16 \\
565,28 \\
612,00 \\
472,84 \\
452,67 \\
654,84 \\
621,76 \\
576,72 \\
496,17\end{array}$ & $\begin{array}{l}37,2 \\
37,5 \\
37,7 \\
37,4 \\
37,4 \\
37,3 \\
37,6 \\
37,4 \\
37,3\end{array}$ & $\begin{array}{l}29 \\
20 \\
20 \\
20 \\
20 \\
20 \\
20 \\
20 \\
20\end{array}$ & $\begin{array}{l}420,88 \\
405,70 \\
773,64 \\
511,45 \\
491,46 \\
728,88 \\
589,43 \\
524,17 \\
430,13\end{array}$ \\
\hline Summe: & 10520,324 & 707,08 & 189 & 10230,272 \\
\hline Mittel: & 556,63 & 37,41 & & 541,28 \\
\hline
\end{tabular}


Aus Generaltabelle I und II folgt:

Sauerstoffverbranch pro Kilo und Stunde bei $380,33 \mathrm{C} .=581,09 \mathrm{ccm}$ Sanerstoffverbranch pro Kilo und Stunde bei $37^{\circ}, 41 \mathrm{C} .=556,63 \mathrm{ccm}$

Also Abnahme für $00,92 \mathrm{C} .=24,46 \mathrm{~cm}$ oder 26,6 cem für $1^{0} \mathrm{C}$. entsprechend $4,5 \%$.

Kohlensäureproduction pro Kilo und Stunde bei $380,29 \mathrm{C} .=570,80 \mathrm{cem}$ Kohlensäureproduction proKilo und Stunde bei $37^{\circ}, 41 \mathrm{C} .=541,28 \mathrm{ccm}$

Also Abnahme für $0^{0}, 88 \mathrm{C} .=29,52$

oder $33 \mathrm{cem}$ für $10 \mathrm{C}$. entsprechend $5,8 \%$.

\section{Generaltabelle III.}

\begin{tabular}{|c|c|c|c|c|}
\hline $\begin{array}{l}\text { Versuchs- } \\
\text { nummer. }\end{array}$ & $\begin{array}{l}\text { Sauerstoff } \\
\text { in } \mathrm{ccm} .\end{array}$ & $\begin{array}{l}\text { Temperatur } \\
\text { in Recto (C.). }\end{array}$ & $\begin{array}{c}\text { Dauer des Ver- } \\
\text { suches in Min. }\end{array}$ & $\begin{array}{l}\text { Kohlensäure } \\
\text { in ccm. }\end{array}$ \\
\hline 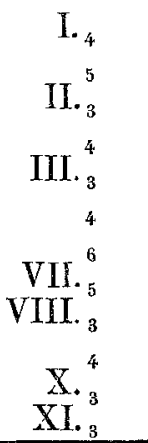 & $\begin{array}{l}413,18 \\
299,79 \\
496,56 \\
510,05 \\
377,92 \\
346,65 \\
202,58 \\
471,13 \\
425,84 \\
271,79 \\
518,84 \\
343,44\end{array}$ & $\begin{array}{l}32,5 \\
30,7 \\
32,3 \\
31,6 \\
31,4 \\
30,5 \\
28,8 \\
30,8 \\
33,1 \\
29,3 \\
32,9 \\
32,2\end{array}$ & $\begin{array}{l}35 \\
35 \\
20 \\
20 \\
20 \\
20 \\
20 \\
20 \\
20 \\
20 \\
20 \\
20\end{array}$ & $\begin{array}{l}440,20 \\
303,27 \\
520,41 \\
460,67 \\
354,62 \\
296,34 \\
366,38 \\
365,79 \\
428,38 \\
296,37 \\
414,26 \\
371,82\end{array}$ \\
\hline Summe: & 10424,995 & 847,0 & 270 & 10352,225 \\
\hline$\overline{\text { Mittel: }}$ & 386,11 & 31,37 & & 383,41 \\
\hline
\end{tabular}

Respiratorischer Quotient 0,99.

Aus Generaltabelle II und III folgt:

Sauerstoffverbrauch pro Kilo und Stunde bei $370,41 \mathrm{C} .=556,63 \mathrm{ccm}$ Sauerstoffverbrauch pro Kilo und Stunde bei $310,37 \mathrm{C} .=386,11 \mathrm{~cm}$

Also Abnahme für $6^{0}, 04 \mathrm{C} .=170,52 \mathrm{ccm}$ oder $28,2 \mathrm{cem}$ für $1^{0} \mathrm{C}$. entsprechend $5,1 \%$. Kohlensäureproduction pro Kilo und Stunde bei $37^{\circ}, 41 \mathrm{C} .=541,28 \mathrm{ccm}$ Kohlensäureproduction pro Kilo und Stunde bei $31^{\circ}, 37 \mathrm{C} .=383,41 \mathrm{cem}$

Also Abnahme für $6^{0}, 04 \mathrm{C}=157,87 \mathrm{~cm}$ oder $26,1 \mathrm{cem}$ für $10 \mathrm{C}$. entsprechend $4,8 \%$. 
Ueber Oxydation im Warmblüter bei subnormalen Temperaturen. 395

Generaltabelle IV.

\begin{tabular}{|c|c|c|c|c|}
\hline $\begin{array}{l}\text { Versuchs- } \\
\text { nummer. }\end{array}$ & $\begin{array}{l}\text { Sauerstoff } \\
\text { in ccm. }\end{array}$ & $\begin{array}{l}\text { Temperatur } \\
\text { in Recto (C.). }\end{array}$ & $\begin{array}{l}\text { Dauer des Ver- } \\
\text { suches in Min. }\end{array}$ & $\begin{array}{l}\text { Kohlensäure } \\
\text { in ccm. }\end{array}$ \\
\hline 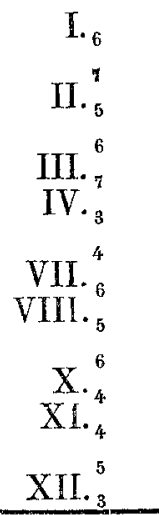 & $\begin{array}{l}211,83 \\
276,44 \\
161,15 \\
183,38 \\
192,19 \\
235,08 \\
175,61 \\
303,40 \\
227,38 \\
220,84 \\
231,03 \\
222,69 \\
196,51 \\
208,36 \\
\end{array}$ & $\begin{array}{l}26,3 \\
25,0 \\
26,2 \\
25,6 \\
27,0 \\
27,6 \\
26,6 \\
26,7 \\
25,5 \\
24,5 \\
27,4 \\
27,1 \\
26,2 \\
24,9 \\
\end{array}$ & $\begin{array}{l}20 \\
25 \\
20 \\
20 \\
20 \\
20 \\
20 \\
20 \\
25 \\
20 \\
20 \\
25 \\
20 \\
20 \\
\end{array}$ & $\begin{array}{l}260,43 \\
178,58 \\
211,18 \\
178,73 \\
101,02 \\
234,82 \\
184,37 \\
223,27 \\
269,99 \\
160,32 \\
245,24 \\
229,46 \\
106,27 \\
220,42 \\
\end{array}$ \\
\hline Summe: & 6455,035 & 772,0 & 295 & 5947,239 \\
\hline Mittel: & 218,82 & 26,17 & - & 201,60 \\
\hline
\end{tabular}

Aus Generaltabelle III und IV folgt:

Sauerstoffverbrauch pro Kilo und Stunde bei $31^{0}, 37 \mathrm{C} .=386,11 \mathrm{ccm}$ Sauerstoffverbrauch pro Kilo und Stunde bei $26^{\circ}, 17 \mathrm{C} .=218,82 \mathrm{~cm}$

Also Abnahme für $5^{0}, 20 \mathrm{C}=167,29 \mathrm{ccm}$ oder 32,2 cem für $1^{0} \mathrm{C}$. entsprechend $8,3 \%$.

Kohlensäureproduction pro Kilo und Stunde bei $31^{0}, 37 \mathrm{C} .=383,41 \mathrm{ccm}$ Kohlensäureproduction pro Kilo und Stunde bei $26^{\circ}, 17 \mathrm{C}=201,60 \mathrm{~cm}$

Also Abnahme für $5^{0}, 20 \mathrm{C}=181,81 \mathrm{ccm}$ oder 34,9 ecm für $1^{0} \mathrm{C}$. entsprechend $9,1 \%$.

Generaltabelle V.

\begin{tabular}{|c|c|c|c|c|}
\hline $\begin{array}{l}\text { Versuchs- } \\
\text { nummer. }\end{array}$ & $\begin{array}{l}\text { Sauerstoff } \\
\text { in } \mathrm{ccm} \text {. }\end{array}$ & $\begin{array}{l}\text { Temperatur } \\
\text { in Recto (C.). }\end{array}$ & $\begin{array}{l}\text { Dauer des Ver- } \\
\text { suches in Min. }\end{array}$ & $\begin{array}{l}\text { Kohlensäure } \\
\text { in ccm. }\end{array}$ \\
\hline $\begin{array}{r}\mathrm{VII}{ }^{\mathrm{X}} \\
{ }_{5} \\
\mathrm{XII}_{4}{ }_{4}\end{array}$ & $\begin{array}{r}79,46 \\
345,89 \\
143,80 \\
156,48 \\
\end{array}$ & $\begin{array}{l}23,2 \\
23,2 \\
22,5 \\
23,4 \\
\end{array}$ & $\begin{array}{l}20 \\
20 \\
20 \\
20\end{array}$ & $\begin{array}{l}202,77 \\
185,26 \\
149,69 \\
175,27\end{array}$ \\
\hline Summe: & 725,63 & 92,3 & 80 & 712,99 \\
\hline Mittel: & 181,41 & 23,07 & & 178,25 \\
\hline
\end{tabular}


Aus Generaltabelle IV and V folgt:

Sauerstoffverbrauch pro Kilo und Stunde bei $26^{\circ}, 17 \mathrm{C} .=218,82 \mathrm{ccm}$ Sauerstoffverbrauch pro Kilo und Stunde bei $23^{\circ}, 07$ C. $=181,41 \mathrm{ccm}$

Also Abnahme für $3^{0}, 10 \mathrm{C} .=37,41 \mathrm{cem}$ oder $12,1 \mathrm{cem}$ für $1^{0} \mathrm{C}$. entsprechend $5,5 \%$.

Kohlensäureproduction pro Kilo und Stunde bei $26^{\circ}, 17 \mathrm{C} .=201,60 \mathrm{~cm}$ Kohlensäureproduction pro Kilo und Stunde bei $23^{\circ}, 07 \mathrm{C} .=178,25 \mathrm{ccm}$

Also Abnahme für $3^{0}, 10 \mathrm{C}=23,35 \mathrm{ccm}$ oder $7,5 \mathrm{ccm}$ für $10 \mathrm{C}$. entsprechend $3,7 \%$. Generaltabelle VI.

\begin{tabular}{c||c|c||c||c}
\hline $\begin{array}{c}\text { Versuchs- } \\
\text { nummer. }\end{array}$ & $\begin{array}{c}\text { Sauerstoff } \\
\text { in ccm. }\end{array}$ & $\begin{array}{c}\text { Temperatur } \\
\text { in Recto (C.). }\end{array}$ & $\begin{array}{c}\text { Dauer des Ver- } \\
\text { suches in Min. }\end{array}$ & $\begin{array}{c}\text { Kohlensäure } \\
\text { in ccm. }\end{array}$ \\
\hline VIII. & 190,64 & 29,5 & 30 & 193,15 \\
X. & 216,90 & 28,9 & 20 & 184,24 \\
8 & 230,80 & 32,8 & 25 & 258,17 \\
\hline Summe: & 1582,72 & 228,3 & 75 & 1468,355 \\
\hline Mittel: & 211,03 & 30,44 & & 195,78 \\
\multicolumn{5}{c|}{ Respiratorischer Quotient $0,93}$.
\end{tabular}

Aus Generaltabelle V und VI folgt:

Sauerstoffverbrauch pro Kilo und Stunde bei $23^{\circ}, 07 \mathrm{C} .=181,41 \mathrm{cem}$ Sauerstoffverbrauch pro Kilo und Stunde bei $30^{\circ}, 44 \mathrm{C} .=211,03 \mathrm{ccm}$ Also Zunahme für $70,37 \mathrm{C} .=29,62 \mathrm{ccm}$ oder 4,0 cem für $1^{0} \mathrm{C}$. entsprechend $2,2 \%$.

Kohlensäureproduction pro Kilo und Stunde bei $23^{n}, 07 \mathrm{C} .=178,25 \mathrm{cem}$ Kohlensäureproduction pro Kilo und Stunde bei $30^{\circ}, 44 \mathrm{C} .=195,78 \mathrm{ccm}$

Also Zunahme für $7^{0}, 37 \mathrm{C} .=17,53 \mathrm{cem}$ oder $2,4 \mathrm{ccm}$ für $1^{\circ} \mathrm{C}$. entsprechend $1,4 \%$. Generaltabelle VII.

\begin{tabular}{|c|c|c|c|c|}
\hline $\begin{array}{l}\text { Versuchs- } \\
\text { nummer. }\end{array}$ & $\begin{array}{l}\text { Sauerstoff } \\
\text { in ecm. }\end{array}$ & $\begin{array}{l}\text { Temperatur } \\
\text { in Recto (C.). }\end{array}$ & $\begin{array}{l}\text { Dauer des Ver- } \\
\text { suches in Min. }\end{array}$ & $\begin{array}{l}\text { Kohlensäure } \\
\text { in ccm. }\end{array}$ \\
\hline IV. $\mathrm{s}$ & 497,36 & 36,4 & 25 & \\
\hline & 492,10 & 36,7 & 30 & 497,46 \\
\hline $\mathrm{XII}_{5}$ & 403,86 & 36,2 & 20 & 391,65 \\
\hline & 397,26 & 36,0 & 20 & 392,22 \\
\hline Summe: & 4321,94 & 345,5 & $95(70)$ & 3060,66 \\
\hline Mittel : & 454,94 & für $\quad \begin{array}{c}0: 36,37 \\
, \mathrm{CO}_{2}: 36,36\end{array}$ & & 437,24 \\
\hline
\end{tabular}

Respiratorischer Quotient 0,96. 
Ueber Oxydation im Warmblüter bei subnormalen Temperaturen. 397

Aus Generaltabelle VI und VII folgt:

Sanerstoffverbrauch pro Kilo und Stunde bei $30^{\circ}, 44 \mathrm{C} .=211,03 \mathrm{ccm}$ Sauerstoffverbrauch pro Kilo und Stunde bei $36^{\circ}, 37 \mathrm{C} .=454,94 \mathrm{cem}$

Also Zunahme für $5^{0}, 93 \mathrm{C} .=243,91 \mathrm{cem}$ oder $41,1 \mathrm{ccm}$ für $1^{0} \mathrm{C}$. entsprechend $19,5 \%$.

Kohlensäureproduction pro Kilo und Stunde bei $30^{\circ}, 44 \mathrm{C} .=195,78 \mathrm{ccm}$ Kohlensäureproduction pro Kilo und Stunde bei $36^{\circ}, 36 \mathrm{C} .=437,24 \mathrm{cem}$

Also Zunahme für $5^{0,92 \mathrm{C} .}=241,46 \mathrm{~cm}$ oder $40,7 \mathrm{ccm}$ für $1^{0} \mathrm{C}$. entsprechend $20,8 \%$.

Vereinigen wir schliesslich die 7 Reihen der Mittelwerthe in eine Tabelle.

\section{Generaltabelle VIII}

abgeleitet aus den Generaltabellen I-VII.

\begin{tabular}{|c|c|c|c|}
\hline $\begin{array}{c}\text { Temperatur } \\
\text { in Recto }\left({ }^{\circ} \mathrm{C} .\right) \text {. }\end{array}$ & \begin{tabular}{|} 
Sauerstoffver- \\
brauch pro Kilo \\
und Stunde in ccm \\
(auf $0^{\circ} \mathrm{C}$. und $0 \mathrm{~m}, 76$ \\
Hg reducirt.)
\end{tabular} & $\begin{array}{l}\text { Kohlensäurebil- } \\
\text { dung pro Kilo und } \\
\text { Stunde in ccm (auf } \\
0^{\circ} \text { C. und } 0,{ }^{\text {m } 76} \\
\text { Hg reducirt.) }\end{array}$ & $\begin{array}{c}\text { Respiratorischer } \\
\text { Quotient. }\end{array}$ \\
\hline $\begin{array}{r}38,33 \\
\left(\mathrm{CO}_{2}: 38,29\right) \\
37,41 \\
31,37 \\
26,17 \\
23,07 \\
30,44 \\
36,37 \\
\left(\mathrm{CO}_{2}: 36,36\right)\end{array}$ & $\begin{array}{l}581,09 \\
556,63 \\
386,11 \\
218,82 \\
181,41 \\
211,03 \\
454,94\end{array}$ & $\begin{array}{l}570,80 \\
541,28 \\
383,41 \\
201,60 \\
178,25 \\
195,78 \\
437,24\end{array}$ & $\begin{array}{l}0,98 \\
0,97 \\
0,99 \\
0,92 \\
0,98 \\
0,93 \\
0,96\end{array}$ \\
\hline
\end{tabular}

Hierans folgt: 


\begin{tabular}{|c|c|c|c|c|}
\hline $\begin{array}{l}\text { Temperatur- } \\
\text { grenzen }\left({ }^{0} \mathrm{C} .\right) \text {. }\end{array}$ & \begin{tabular}{|c} 
Mittlere Ab- \\
nahme des \\
Sauerstoffver- \\
brauchs pro Kilo \\
und Stunde bei \\
Abnahme dex \\
Körpertempera- \\
tur unter die \\
Norm, in ccm, \\
für 1 C. $^{\circ}$. \\
berechnet.
\end{tabular} & \begin{tabular}{|c|} 
Mittlere Ab- \\
nahme der \\
Kohlensäureab- \\
gabe pro Kilo \\
und Stunde bei \\
Abnahme der \\
Körpertempera- \\
tur unter die \\
Norm, in ccm, \\
für 1" C. \\
berechnet.
\end{tabular} & \begin{tabular}{||c|} 
Procentische \\
Abnahme \\
des Sauer- \\
stoffver- \\
brauchs bei \\
Abnahme \\
der Körper- \\
temperatur \\
unter die \\
Norm \\
um $1^{\circ} \mathrm{C}$.
\end{tabular} & $\begin{array}{l}\text { Procentische } \\
\text { Abnahme } \\
\text { der Kohlen- } \\
\text { säurepro- } \\
\text { duction bei } \\
\text { Abnahme } \\
\text { der Körper- } \\
\text { temperatur } \\
\text { unter die } \\
\text { Norm } \\
\text { am } 1^{\circ} \mathrm{C} \text {. }\end{array}$ \\
\hline \multirow[t]{2}{*}{$\begin{array}{c}38^{0}, 33-37^{0}, 41 \\
\left(38^{\circ}, 29-37^{0}, 41\right) \\
37^{\circ}, 41-31^{\circ}, 37 \\
31^{\circ}, 37-26^{\circ}, 17 \\
26^{\circ}, 17-23^{0}, 07\end{array}$} & $\begin{array}{l}26,6 \\
\overline{28,2} \\
32,2 \\
12,1\end{array}$ & $\begin{array}{r}-- \\
33,0 \\
26,1 \\
34,9 \\
7,5\end{array}$ & $\begin{array}{l}4,5 \% \\
5,1 \% \\
8,3 \% \\
5,5 \%\end{array}$ & $\begin{array}{l}\overline{5,8 \%} \\
4,8 \% \\
9,1 \% \\
3,7 \%\end{array}$ \\
\hline & \begin{tabular}{|c|} 
Mittlere Stei- \\
gerung des \\
Sauerstoffver- \\
brauchs pro \\
Kilo und Stunde \\
bei allmähliger \\
Steigerung der \\
Körpertempera- \\
tur nach ge- \\
schehener Ab- \\
kühlung, für \\
10 $\mathrm{C}$. berechnet.
\end{tabular} & \begin{tabular}{|c|} 
Mittlere Stei- \\
gerung der \\
Kohlensäureab- \\
gabe pro Kilo \\
und Stunde bei \\
allmähliger Stei- \\
gerung der Kör- \\
pertemperatur \\
nach geschehe- \\
ner Ablüulung; \\
für $\mathbf{1}^{\circ} \mathrm{C}$. \\
berechnet.
\end{tabular} & $\begin{array}{c}\text { Wie oben } \\
\text { procentische } \\
\text { Steigerung. }\end{array}$ & $\begin{array}{l}\text { Wie oben } \\
\text { procentische } \\
\text { Steigerung. }\end{array}$ \\
\hline $\begin{array}{c}23^{0}, 07-30^{0}, 44 \\
30^{\circ}, 44-36^{0}, 37 \\
\left(30^{\circ}, 44-36^{0}, 36\right)\end{array}$ & $\begin{array}{r}4,0 \\
41,1 \\
-\end{array}$ & $\frac{2,4}{40,7}$ & $\begin{array}{r}2,2 \% \\
19,5 \% \\
\end{array}$ & $\begin{array}{l}1,4 \% \\
20, \overline{8} \%\end{array}$ \\
\hline
\end{tabular}

Das Ergebniss der vorstehenden Untersuchungen ist durch beiliegende Oxydationscurve ausgedrückt.

Zum Schlusse sage ich Herrn Dr. Dittmar Finkler meinen Dank für dic vielfache Unterstiitzung, die derselbe bei Ausführung der oben beschriebenen Versuche in der liebenswürdigsten und zuvorkommendsten Weise mir geleistet hat. 


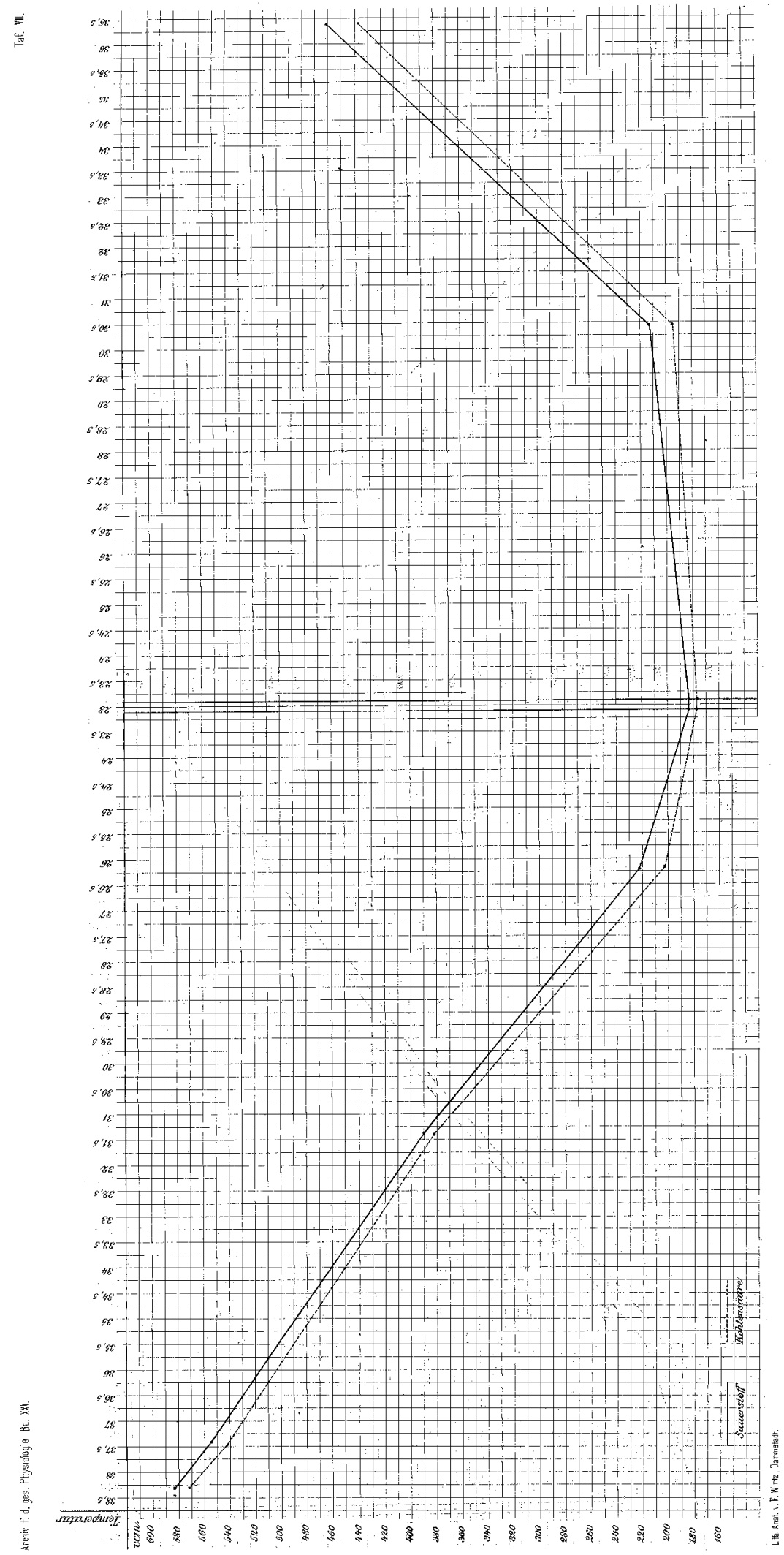

